\title{
Experimental Investigation and Numerical Simulation on Interaction Process of Plasma Jet and Working Medium
}

\author{
Yong-gang Yu, Na Zhao, Shan-heng Yan and Qi Zhang \\ Nanjing University of Science and Technology \\ China
}

\section{Introduction}

The interaction between the plasma jet and medium has been applied in the field as aerospace and armament. In order to meet the requirement of the spacecraft's orbital adjustment, gravitation compensate, position maintenance, orbital maneuver and the attitude control, many kinds of micro propulsion system, such as: micro electrical propulsion, micro cold air propulsion, micro laser propulsion, pulse plasma propulsion (Robert, 2003) and so on have been studied in many countries. The operation principle of the pulse plasma propulsion is as follows: the electric arc plasma forms by loading the electric energy at two ends of the capillary. The capillary plasmas with high temperature and pressure are then produced as the capillary wall can be burned by the electric arc. The plasma ejects out through the nozzle and pushes the chamber moving forward. In modern hypervelocity launching technique, the liquid propellant electrothermal-chemical technology (LPETC) is one of the effective one and develops well. The LPETC propulsion technology is a new propulsion technique to ignite the propellant by the high pressure and high temperature pulse plasma jet produced by capillary discharge. Accordingly, performance of the plasma jet and the interaction between the plasma jet and the medium (Nusca et al., 2001) is one of the key problems in plasma propulsion and the electrothermalchemical launching technique. Lots of works have been done according the related field by many scholars. Taylor M J studied the free expansion processes of the plasma in the atmosphere as the discharge energy is 30KJ (Taylor, 2001). The distributions of parameters as the temperature and pressure have been got. The free expansion characteristics of the plasma jet have been studied by Kim J U et al. as the discharge energy is $3.1 \mathrm{KJ}$ (Kim \& Suk, 2002). The temperature and density distribution of plasma and the shock wave structure of the incompletion expansion jet have been got. The effects of discharge pulse length on the characteristics of plasma jet impacting the plate as the discharge energy is $3 \mathrm{KJ}$ has been studied by Lang-Mann Chang et al.(Chang, Harward, 2007). Guo H B et al. studied the discharge characteristics in the capillary (Guo, Liu, et al., 2007). Zhang Q et al. studied the expansion characteristics of the plasma jet in atmosphere as the discharge energy is less than 100J (Zhang et al., 2009). Wilsion D E et al. proposed the plasma jet axisymmetry unstable model (Wilsion \& Kim, 1999). The plasma jet is treated as over expansion supersonic speed instantaneous jet and the development of expansion wave and Mach disc have been 
simulated. The interaction between the plasma jet and liquid has been studied by Kuo K K et al. by high speed camera and pulse X-ray imaging technology (Kuo et al., 1990). The expansion processes of Taylor cavity formed as the plasma jets into the liquid and the intensity distribution law of plasma have been got. Arensburg A studied the continuous expansion processes of the plasma jet in water by shadow imaging technology (Arensburg, 1993). The jet speeds, the mass flowrate entrainment by the liquid at two phase interface are studied quantitatively. The droplets' formation process due to the liquid entrainment was also studied. Zhou Y H studied the interaction characteristics between the plasma jet and the liquid medium in the cylinder inspection chamber(Zhou et al., 2003). Yu Y G et al. studied the interaction between the plasma jet and the liquid medium both in cylinder and the stepped-wall inspection chamber (Yu et al., 2009). The effects of the boundary shape on the expansion characteristics of plasma jet have been studied.

In this paper, the expansion characteristics of plasma jet in atmosphere and the interaction properties between the plasma jet and the liquid medium on the small discharge energy condition have been studied. The effects of the discharge voltage, nozzle diameter and the multilevel steps boundary shape of the stepped-wall chamber on the expansion characteristics of Taylor cavity caused by plasma jet have been mainly discussed. Twodimensional axisymmetry mathematic model of the interaction between plasma jet and the liquid medium has been proposed based on the experiment. The expansion processes of plasma jet on the unsteady state condition have be simulated. The distribution performances of the pressure, velocity and temperature in flow field have been got.

\section{Experiment apparatus of plasma jet generator}

The simulated experiment apparatus are composed of plasma generator, pulse power source and so on as shown in figure 1. The plasma generator is made up of polythene capillary, electrodes, metal detonating cord, the metal sealed film on the cathode, the insulator and the metal shell outside the capillary and so on. The pulse power supply is composed of the pulse forming network (PFN) whose energy is stored by capacitor as shown in figure 2 .

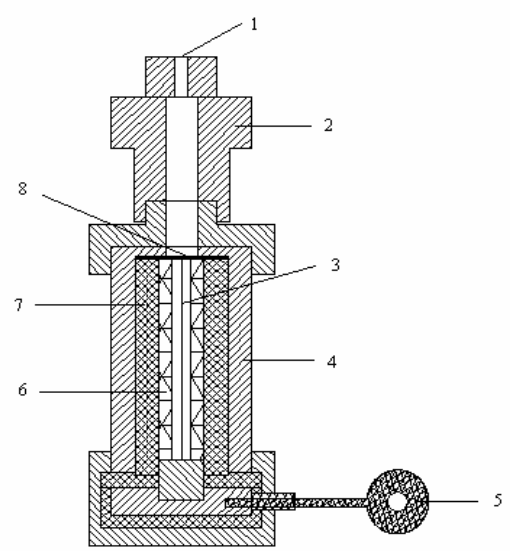

1- nozzle; 2- joining; 3- exploding wire; 4- steel shell; 5- anode; 6- polyethylene capillary; 7- insulator; 8- copper film

Fig. 1. Schema of the experiment apparatus 


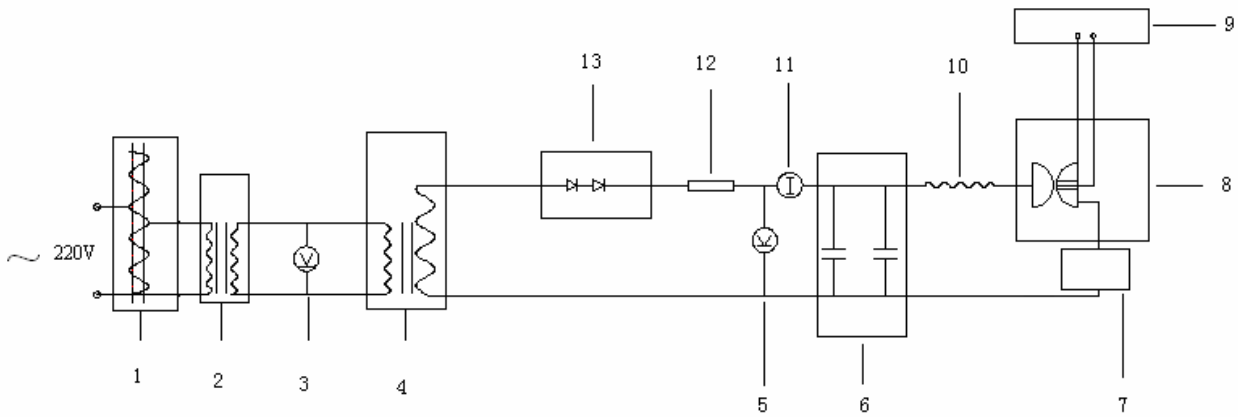

1-autotransformer; 2-isolating transformer; 3-AC voltmeter; 4-step-up transformer; 5-DC voltmeter; 6capacitor bank; 7-plasma generator; 8-discharge switch; 9-discharge trigger; 10-damping resistor; 11charging ammeter; 12-current-limting resistor; 13-rectifier stack

Fig. 2. Pulse-forming network setup

The capillary as the load of the discharge loop is connected to the pulse power supply. There is a hole in the cathode. The polythene pipe is ablated by the high power discharge. The plasma forms and flows into the nozzle through the hole in the cathode. The anode is connected to the high voltage output of the pulse power source and keeps sealed. The cathode connects earth by the body of the apparatus. The output intensity of the plasma is adjusted by changing the capacitors' discharge voltage and the discharge loop's parameters. The cathode of the plasma generator is sealed by the metal film before the experiment. The jet is started until the pressure in the capillary increasing to a threshold value in case of discharge is terminated as the electric arc is break-off too early. The diameter of the nozzle and the thickness of the film can be changed according the need of the experiment.
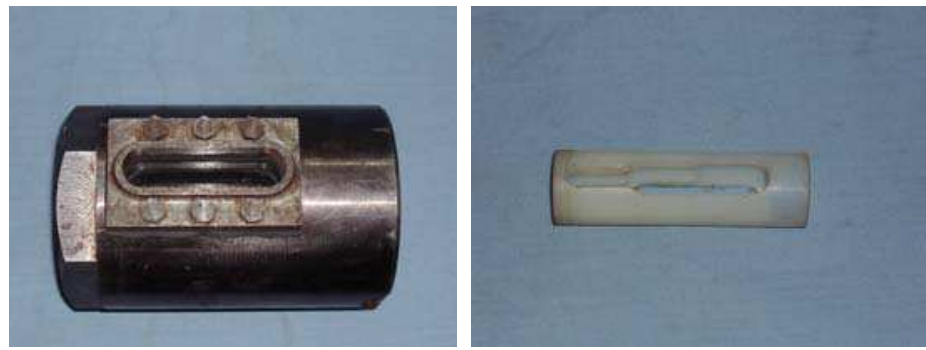

Fig. 3. Decomposition schema of the stepped-wall chamber

An inspection chamber is set up at the exit of the nozzle in order to study the interaction characteristics between the plasma jet and liquid medium visible. The inspection chamber is a cylinder liquid container and the 4 levels stepped-wall chamber is inserted into it, as shown in figure 3. There are inspection window at two opposite sides of the chamber near the nozzle and the window is sealed by chemical method. In order to eliminate the effects of the gravity, the experiment apparatus is placed upright and the plasma is injected upward. In addition, the top end of the chamber is open-end to the atmosphere in case of the window is broken by the over high pressure forming through the expansion of plasma jet. The high speed camera system is used to record the interaction processes between the plasma jet and the liquid medium. And the pressure in the capillary is measured by the pressure sensor. 


\section{Experiment results of the plasma jet expansion in atmosphere}

Figure 4 shows the typical pictures of the sequence expansion processes of the plasma jet in the atmosphere as the discharge voltage is $2500 \mathrm{~V}$ and the diameter of the nozzle is $4 \mathrm{~mm}$.

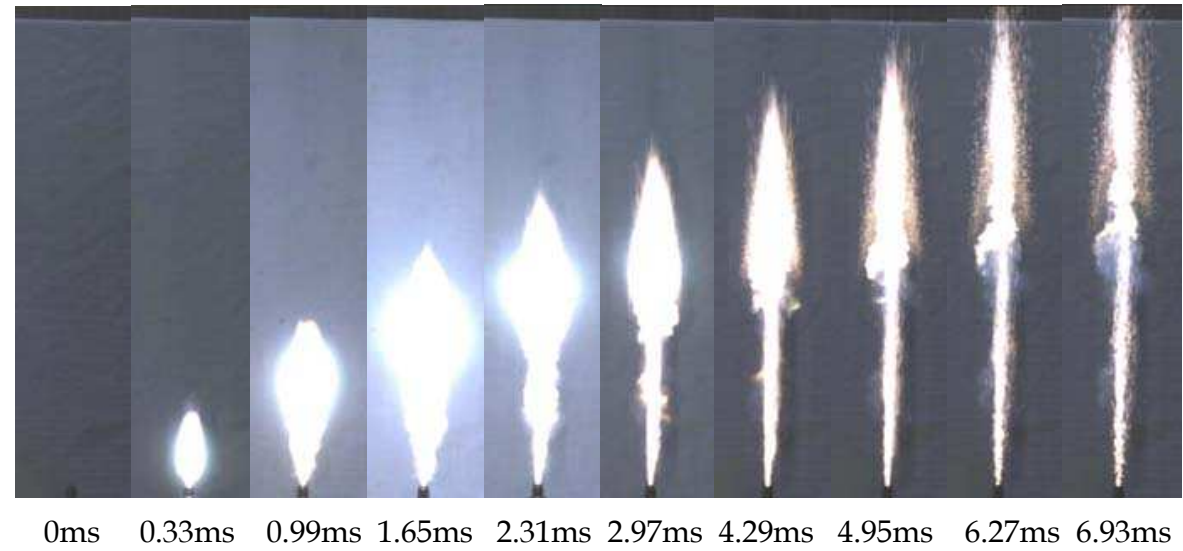

Fig. 4. Sequence processes of the plasma jet in the atmosphere

As shown in the figure, the plasma jet expands both along the axial and radial direction and the axial velocity is larger than the radial one. During the expansion of the plasma jet, the shape of the jet head changes from similar ellipsoid to taper and the jet shape is longer and thinner gradually. As shown in figure, as $t=2.97-6.93 \mathrm{~ms}$, there is intensity turbulence dissipation phenomena as the plasma jet interacts with the atmosphere. At the beginning, the jet head is drape and the turbulence is strengthened and the turbulent mixture region grows as the development of the jet. The brightness of the plasma jet reflects the temperature. During the plasma jet's expansion processes, the brightness of the plasma jet increases and then decay. The jet head is brighter. It indicates that at the initial of the jet, the temperature decreases after increases as the going of the time. And the jet head has a higher temperature.

\subsection{Effects of the discharge voltage on the expansion process of plasma jet}

Figure 5 shows the sequence expansion processes of the plasma jet in the atmosphere as the discharge voltage is $2100 \mathrm{~V}, 2500 \mathrm{~V}$ and $3000 \mathrm{~V}$ respectively and the nozzle diameter is $4 \mathrm{~mm}$. As shown in the figure, the expansion shape is similar at different voltages while the jet intensity is different. The larger is the discharge voltage, the larger is the jet intensity and the jet head's turbulence dissipation is greater. As the discharge voltage increases from $2100 \mathrm{~V}$ to $2500 \mathrm{~V}$, the expansion is strengthened both in the axial and radial direction and the jet is brighter. But there is tiny effect of the voltage on the jet axial expansion as the discharge voltage changes from $2500 \mathrm{~V}$ to $3000 \mathrm{~V}$, while the effect on the radial expansion is obvious. As the discharge voltage is $3000 \mathrm{~V}$, the radial expansion velocity is larger at initial and the turbulence dissipation is intensity in the later period. At the time after $\mathrm{t}=2.64 \mathrm{~ms}$, the mixture region of the plasma and the atmosphere is thick and the boundary is fade. The lightness of the plasma is brightest as the discharge voltage is $3000 \mathrm{~V}$ as shown in the figure. 


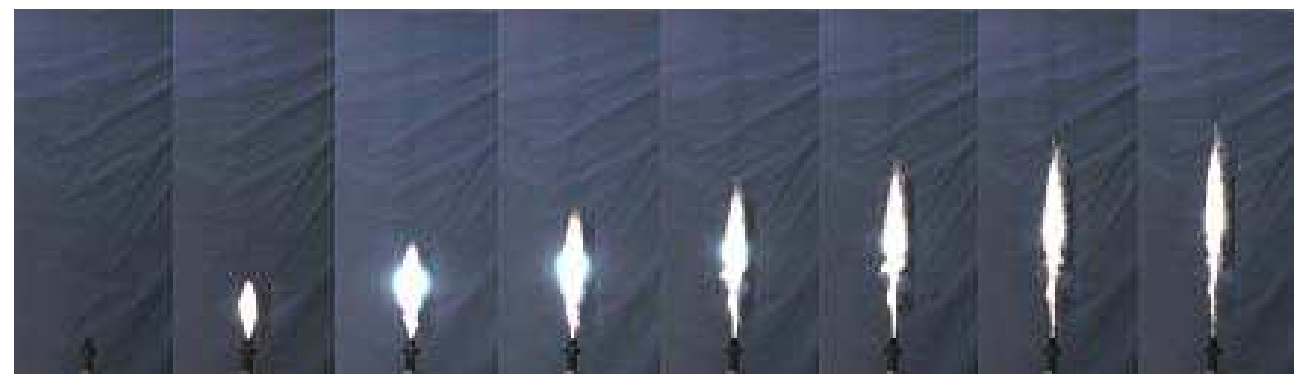

0ms

$0.66 \mathrm{~ms}$

$1.32 \mathrm{~ms}$

$1.98 \mathrm{~ms}$

$2.64 \mathrm{~ms}$

$3.3 \mathrm{~ms}$

$3.96 \mathrm{~ms}$

$4.62 \mathrm{~ms}$

a) $U_{c}=2100 \mathrm{~V}$

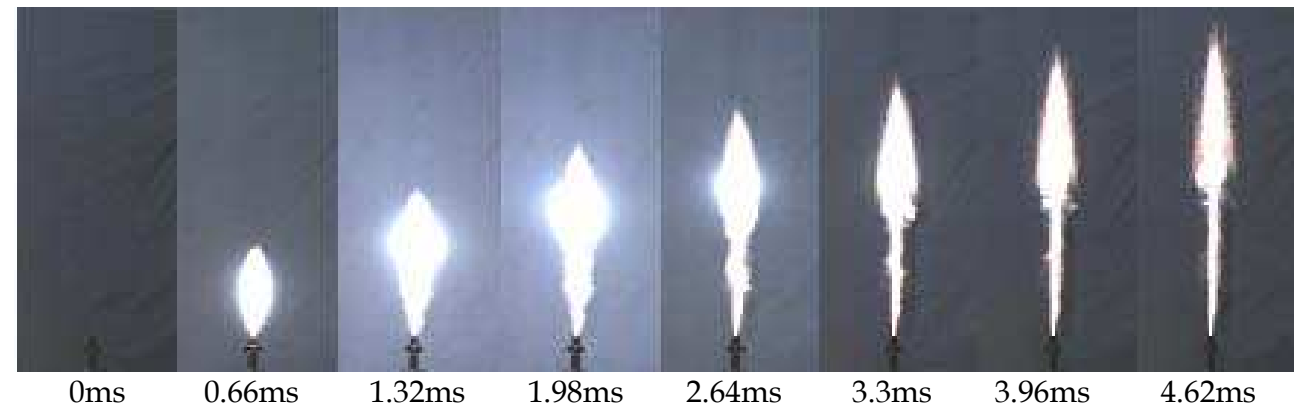

b) $U_{c}=2500 \mathrm{~V}$

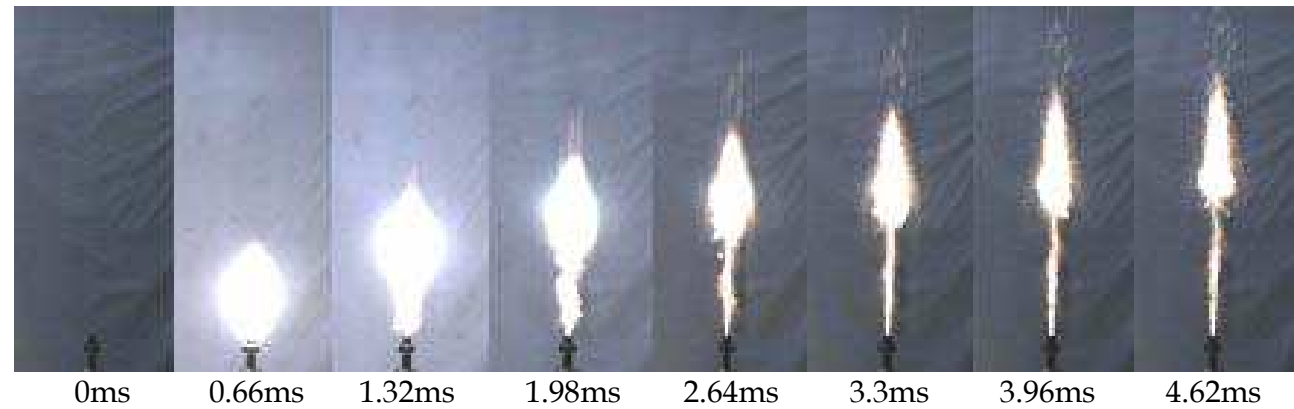

c) $U_{c}=3000 \mathrm{~V}$

Fig. 5. Sequence expansion processes of the plasma jet in the atmosphere at different discharge voltages

The axial and radial expansion displacement of plasma jet can be got from the sequence expansion pictures. And according to the relationship between the expansion displacement and the time, the rules of the expansion velocity changing with time can be handled out. Figure 6 shows the axial $(\mathrm{x})$ and radial $(\mathrm{r})$ expansion displacement changing with the time at different discharge voltages. Figure 7 shows the axial $\left(v_{x}\right)$ and radial $\left(v_{r}\right)$ expansion velocity changing with time at different discharge voltages. 


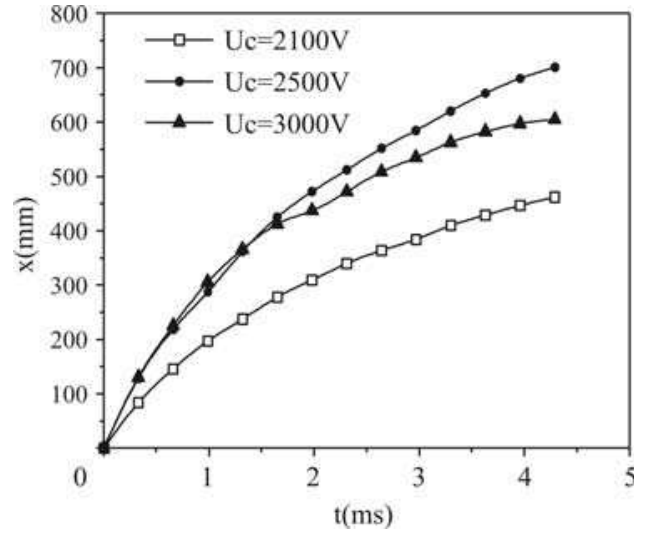

a) $x-t$

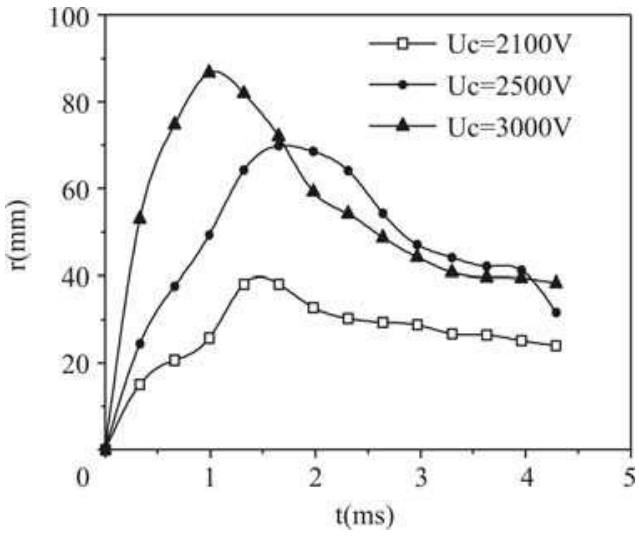

b) $\mathrm{r}-\mathrm{t}$

Fig. 6. The expansion displacement distribution of the plasma jet

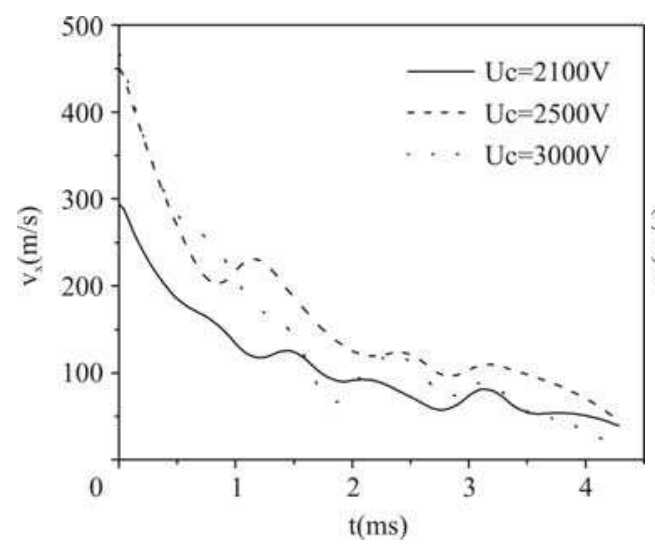

a) $v_{x}-t$

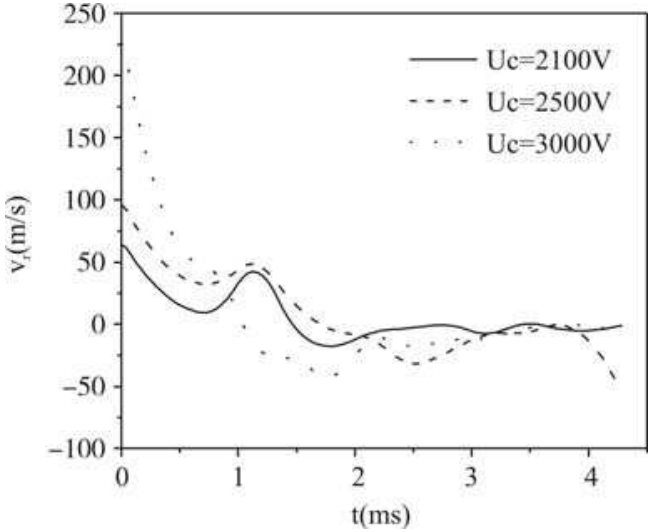

b) $\mathrm{v}_{\mathrm{r}}-\mathrm{t}$

Fig. 7. The expansion velocity distribution of the plasma jet

As shown in figure 6(a), the axial displacement of the plasma increases obviously as the voltage changes from $2100 \mathrm{~V}$ to $2500 \mathrm{~V}$. In the discharge voltage region of $2500 \mathrm{~V}$ to $3000 \mathrm{~V}$, the axial displacement change is small but the radial displacement has a great change. As the discharge voltage is $3000 \mathrm{~V}$, the radial expansion velocity of the plasma jet is greater at the initial expansion, and the turbulence dissipation is higher, the decay of the axial and radial expansion velocity is greater too. The axial and radial expansion displacement as the voltage is $3000 \mathrm{~V}$ may be less than that as the discharge voltage is $2500 \mathrm{~V}$ in the last for the higher dissipation. The axial and radial velocity is decaying as the time goes on and the change curve is fluctuation. The relationships between the axial and radial expansion displacement with the discharge voltage are not monotony. The velocity also does not change with the discharge voltage monotonously. 


\subsection{Effects of the nozzle diameter on the expansion process of plasma jet}

The nozzles with diameter $\left(\mathrm{d}_{0}\right)$ of $1.5 \mathrm{~mm}$ and $2 \mathrm{~mm}$ are adopted to study the effect of the nozzle diameter on the expansion characteristics of the plasma and the discharge voltage is $3000 \mathrm{~V}$. Figure 8 shows the axial displacement of the plasma jet changes with the time at different nozzle diameters.

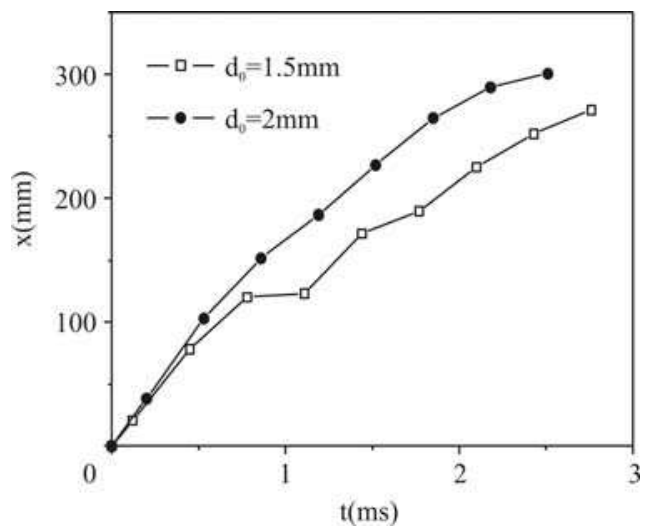

Fig. 8. Changing of the axial expansion displacement of the plasma jet with time at different nozzle diameters

As $\mathrm{t}=0 \sim 0.5 \mathrm{~ms}$, the difference is small between the two curves as can be seen from the figure. And in the stage of $t=0.5 \sim 3 \mathrm{~ms}$, the bigger is the nozzle diameter, the greater is the axial displacement.

\section{Experiment results of the plasma jet expansion in liquid}

The multilevel stepped-wall simulated inspection chamber are adopted to study the interaction properties between the plasma jet and the simulated liquid medium at the cold experiment condition based on the work of Kuo K K(Kuo et al., 1990); Kim H J (Kim \& Hong, 1995); Rott M (Rott \& Artelt, 2005) and so on. The expansion characteristics of Taylor cavity formed by the plasma jet on different conditions can be observed. Some references about the combustion stability of the bulk-loaded liquid propellant controlled by the plasma ignition as can be given in the stepped-wall chamber.

\subsection{Effects of the boundary shape on the expansion process of plasma jet}

The first stage of the stepped-wall chamber is $14 \mathrm{~mm}$ in diameter, and $30 \mathrm{~mm}$ in length. The later three stages are all $30 \mathrm{~mm}$ in length, and each stage's diameter is $6 \mathrm{~mm}$ larger than its former one. Cylindrical chamber is $26 \mathrm{~mm}$ in diameter, and $107 \mathrm{~mm}$ in length. The chamber is full of water, and the plasma is ejected upward. The capacity of the capacitors is $45 \mu \mathrm{f}$, the charging voltage is $2500 \mathrm{~V}$, and the nozzle diameter is $2.5 \mathrm{~mm}$.

The sequential pictures of plasma jet expansion in water in stepped-wall chamber are illustrated in figure 9 .

As can be seen in these pictures, a small, bulb like, bright bubble appears near the nozzle when plasma starts to jet out from nozzle, and the bubble expands downstream further with the plasma and forms the Taylor cavity. These pictures also show that in the expansion processes 
of Taylor cavity, the plasma with high temperature and high luminance illuminates the liquid around Taylor cavity. At the time $t=1 \mathrm{~ms}$, we can see some black dots obviously at the jet head, because the metal plasma produced by metal initiation wire is cooled down into opaque solid particles as the contact with the liquid. And after $\mathrm{t}=2 \mathrm{~ms}$, dark, smog like region appears at the frontal area of the jet. It can be explained as: when the plasma jets into the liquid, KelvinHelmholtz instabilities occur due to the dispatch of velocities at the gas-liquid interface, and the results of plasma jet entrainment to water leads to the liquid break-up, temperature reducing, finally the dark, smog like region forms. Starts from the time $t=2 m s$, there have already been dark dots at the core of the bubble. This phenomenon demonstrates that the cavity can also entrain the surrounding liquid. The analytical results indicate that there is strong heat transfer and mass transfer at the gas-liquid interface.

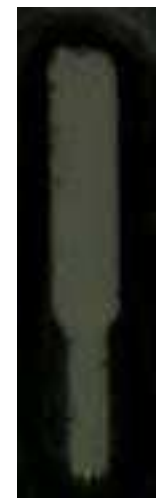

$\mathrm{t}=0 \mathrm{~ms}$

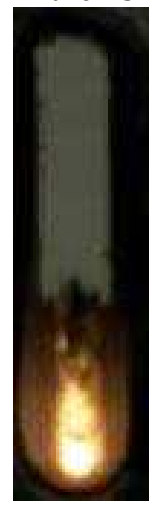

$\mathrm{t}=2.5 \mathrm{~ms}$
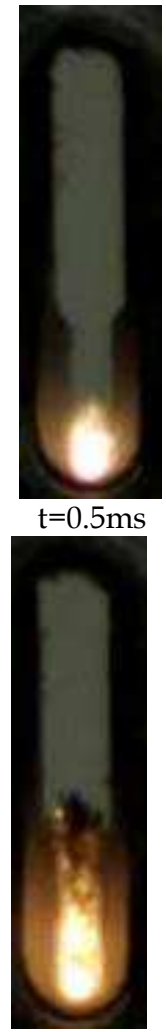

$\mathrm{t}=3 \mathrm{~ms}$

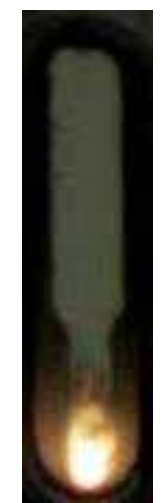

$\mathrm{t}=1 \mathrm{~ms}$

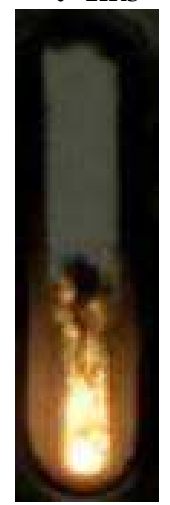

$\mathrm{t}=3.5 \mathrm{~ms}$
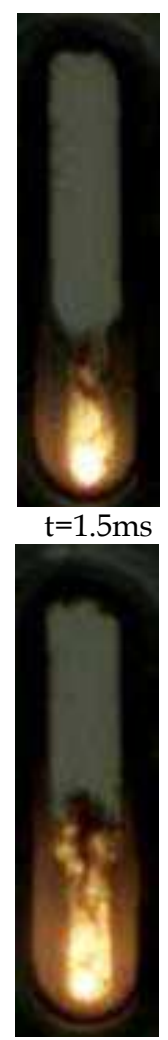

$\mathrm{t}=4 \mathrm{~ms}$

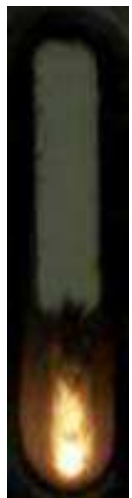

$\mathrm{t}=2 \mathrm{~ms}$

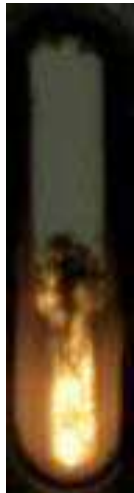

$\mathrm{t}=4.5 \mathrm{~ms}$

Fig. 9. Sequence pictures of plasma jet expansion in stepped-wall chamber

The chamber used in this experiment is stepped-wall chamber, so the radial expansion of Taylor cavity is restricted by the boundary, and the axial velocity is much greater than the radial velocity, as it is shown in figure 9. But in the traditional cylindrical chamber, the radial disturbance should be decreased obviously. So we carried out experiment in cylindrical chamber at the same conditions, i.e. the capacity of capacitors is $45 \mu \mathrm{f}$, the charging voltage is $2500 \mathrm{~V}$, and the nozzle diameter is $2.5 \mathrm{~mm}$. The expansion process of plasma jet is shown in figure 10. 


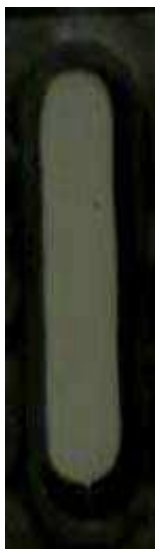

$\mathrm{t}=0 \mathrm{~ms}$

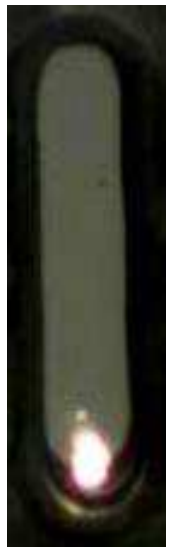

$\mathrm{t}=0.5 \mathrm{~ms}$

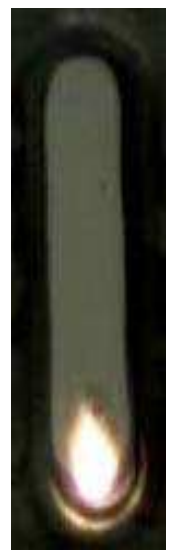

$\mathrm{t}=1 \mathrm{~ms}$

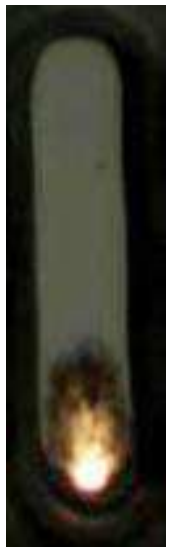

$\mathrm{t}=1.5 \mathrm{~ms}$

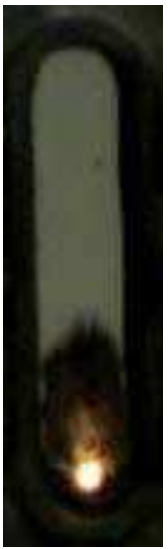

$\mathrm{t}=2 \mathrm{~ms}$

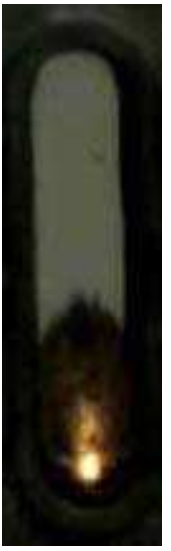

$\mathrm{t}=2.5 \mathrm{~ms}$

Fig. 10. Expansion process of plasma jet in cylindrical chamber

As it is shown in figure 9 and figure 10, the plasma jet is axisymmetric on the whole, somewhat similar to the expansion process of muzzle wave. The initial expansion velocity at the time when the Taylor cavity is formed is larger, and decreased gradually as the Taylor cavity expanding downward. At the same time, the size of Taylor cavity and the expansion velocity is varied with the difference of chamber structure. By comparing these two sets of pictures, we can see that most plasma energy has been exhausted before it reaches the first step (30mm in length). The water in stepped-wall chamber is less than that in cylindrical chamber, the momentum of plasma jet is small, so the axial velocity of plasma jet in steppedwall chamber is greater than that in cylindrical chamber. The plasma jet is interrupted in stepped-wall chamber, but in cylindrical chamber it is not. It is because the boundary of stepped-wall chamber can enhance the radial disturbance to plasma jet. The axial expansion velocity of Taylor cavity can be deduced from the frontal locations of the cavity recorded in the pictures and the corresponding time. Axial velocities of Taylor cavity due to the consecutive expansion of plasma jet in different chambers are compared in figure 11.

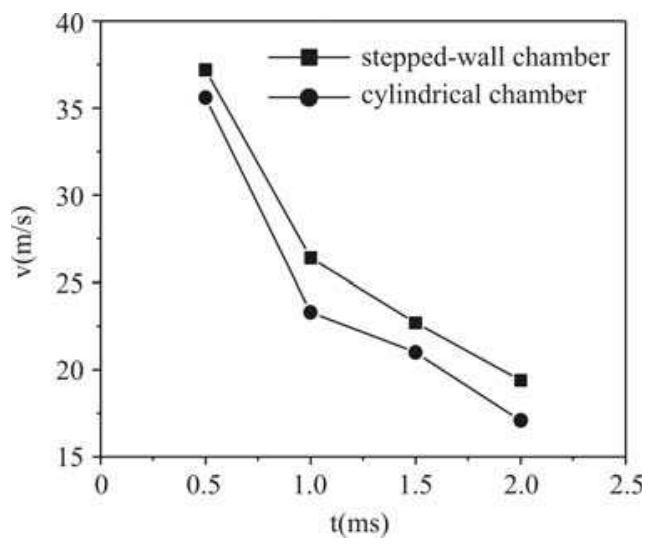

Fig. 11. Comparison of axial velocities of Taylor cavity in two different chambers 


\subsection{Effects of discharge voltage on the expansion process of plasma jet}

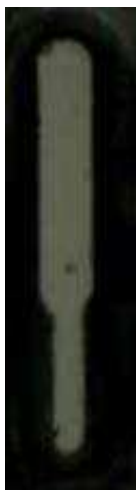

$\mathrm{t}=0 \mathrm{~ms}$

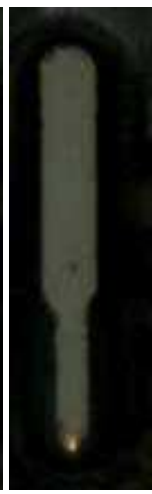

$\mathrm{t}=0.5 \mathrm{~ms}$

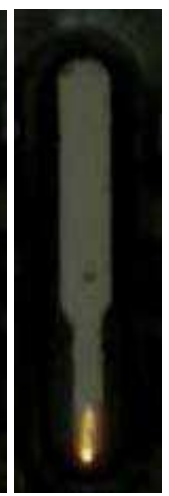

$\mathrm{t}=1 \mathrm{~ms}$

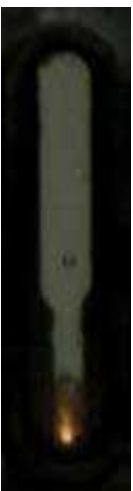

$\mathrm{t}=1.5 \mathrm{~ms}$

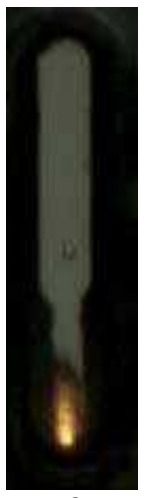

$\mathrm{t}=2 \mathrm{~ms}$

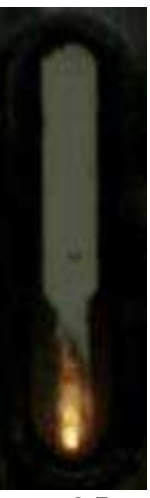

$\mathrm{t}=2.5 \mathrm{~ms}$

a) Discharging voltage $=2000 \mathrm{~V}$

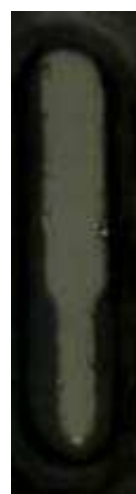

$\mathrm{t}=0 \mathrm{~ms}$
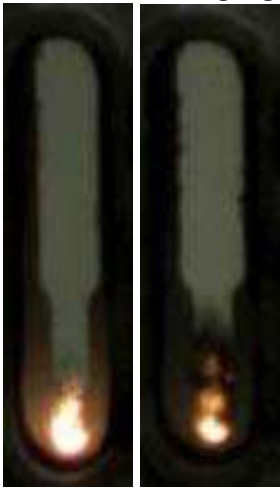

$\mathrm{t}=1 \mathrm{~ms}$
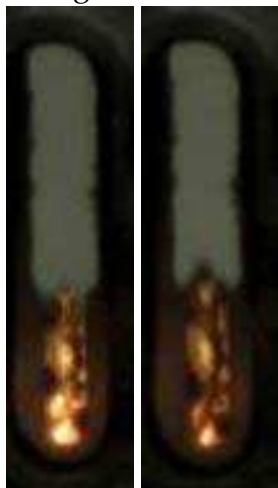

$\mathrm{t}=2 \mathrm{~ms}$

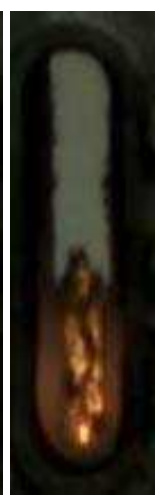

$\mathrm{t}=2.5 \mathrm{~ms}$

b) Discharging voltage $=2300 \mathrm{~V}$

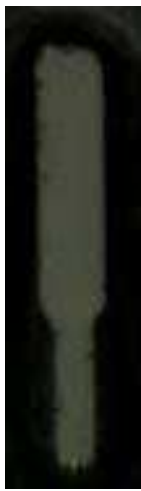

$\mathrm{t}=0 \mathrm{~ms}$

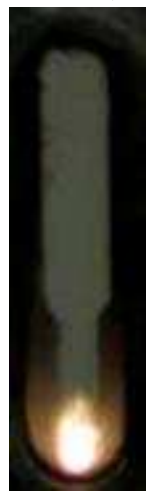

$\mathrm{t}=0.5 \mathrm{~ms}$

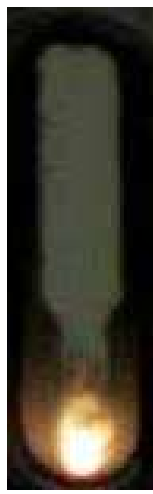

$\mathrm{t}=1 \mathrm{~ms}$

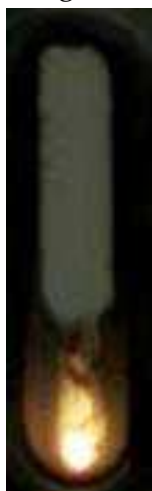

$\mathrm{t}=1.5 \mathrm{~ms}$

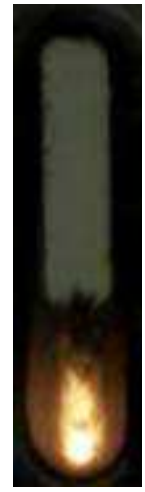

$\mathrm{t}=2 \mathrm{~ms}$

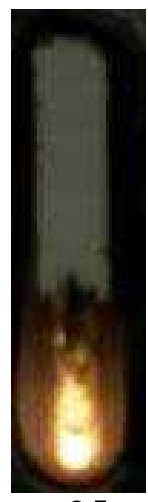

$\mathrm{t}=2.5 \mathrm{~ms}$

c) Discharging voltage $=2500 \mathrm{~V}$

Fig. 12. The sequential pictures of plasma jet expansion in water under different discharging voltages 
In order to discuss the effect of discharging energy on the propagation of plasma jet in stepped-wall chamber, we adjust the discharging voltage to produce plasma jets with different discharging energy, while keeping the structure of stepped-wall chamber and capacity of capacitors unchanged $(45 \mu \mathrm{f})$. The discharging voltages in figure 6 are 2000V, $2300 \mathrm{~V}$ and $2500 \mathrm{~V}$ respectively. Because the conversion efficiency of the pulse power supply is $40 \%$, the resulting discharging energy is $36 \mathrm{~J}$, $48 \mathrm{~J}$ and $56 \mathrm{~J}$ separately. The corresponding propagation processes of plasma jets are illustrated in figure 12(a), 12(b) and 12(c).

The pictures indicate that the lager the discharging energy is the brighter and the bigger the fireball is. The expansion velocity of Taylor cavity in axial direction is greater than that in radial direction as the plasma jet moving upward. When plasma jet develops to a certain degree, a shadow region appears at the jet head, i.e. the luminance is reduced, as the result of liquid vaporization and temperature reducing. The plasma jet is interrupted earlier, and its attenuation is faster while the discharging energy is smaller.

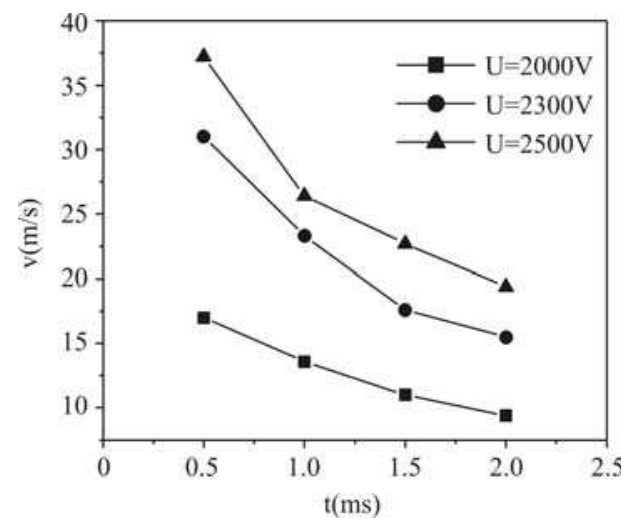

Fig. 13. Changing of the expansion velocity of Taylor cavity with time under different discharging voltages

By measuring the frontal location of Taylor cavity away from the nozzle and its corresponding time, the axial expansion velocity of Taylor cavity can be calculated. As shown in figure 13, the plasma jet velocity has a descending tendency on the whole, and the velocity increases with the increasing of discharging energy at the same time.

\section{Mathematical and physical models of the Interaction process of plasma jet and working medium}

\subsection{Physical model}

According to the constrained expansion character of the plasma jet in stepped-wall chamber, combination with the experimental conditions, the following hypotheses are needed for the physical processes of the jet expansion:

1. The expanding processes of the plasma jet in atmosphere and liquid are the unsteady processes of two-dimensional axial symmetry.

2. Treat the plasma mixture as the ideal gas and without consideration of the chemical reaction between plasma and liquid.

3. Neglect the influence of the secondary factor like electromagnetic force, mass force and the volume force. 
4. The $k-\varepsilon$ model is used to describe the turbulent mixing effect between plasma and liquid in the processes of the plasma jet expansion.

5. The radiation is neglected and the plasma is in partial heat balance condition.

\subsection{Mathematical model}

1. Equation of mass conservation:

The mass conservation equation is as follow:

$$
\frac{\partial \rho}{\partial t}+\frac{\partial(\rho u)}{\partial x}+\frac{\partial(\rho v)}{\partial r}+\frac{\rho v}{r}=0
$$

In which $\mathrm{x}$ is the axial coordinate, $\mathrm{r}$ is the radial coordinate, $\mathrm{u}$ is the axial velocity and $v$ is the radial velocity.

2. Equation of momentum conservation

Equation of axial momentum conservation:

$$
\frac{\partial}{\partial t}(\rho u)+\frac{\partial}{\partial x}(\rho u u)+\frac{\partial}{\partial r}(\rho u v)+\frac{\rho u v}{r}=\frac{1}{r} \frac{\partial\left(r P_{x r}\right)}{\partial r}+\frac{\partial P_{x x}}{\partial x}
$$

Equation of radial momentum conservation:

$$
\frac{\partial}{\partial t}(\rho v)+\frac{\partial}{\partial r}(\rho v v)+\frac{\partial}{\partial x}(\rho v u)+\frac{\rho v v}{r}=\frac{1}{r} \frac{\partial\left(r P_{r r}\right)}{\partial r}+\frac{\partial P_{r x}}{\partial x}
$$

For Newtonian fluid, the stress tensor and the strain rate tensor are listed as follows respectively ( $\mu$ is the dynamic viscosity):

$$
\begin{aligned}
& p_{x x}=-p+2 \mu\left(\varepsilon_{x x}-\frac{1}{3} \operatorname{div} \vec{V}\right), \quad \varepsilon_{x x}=\frac{\partial u}{\partial x} \\
& p_{r r}=-p+2 \mu\left(\varepsilon_{r r}-\frac{1}{3} \operatorname{div} \vec{V}\right), \quad \varepsilon_{r r}=\frac{\partial v}{\partial r} \\
& p_{x r}=p_{r x}=2 \mu \varepsilon_{x e r}, \quad \varepsilon_{r x}=\varepsilon_{x r}=\frac{1}{2}\left(\frac{\partial u}{\partial x}+\frac{\partial v}{\partial r}\right)
\end{aligned}
$$

3. Equation of energy conservation:

$$
\begin{aligned}
\frac{\partial(\rho E)}{\partial t}+\frac{1}{r} \frac{\partial(r \rho v E)}{\partial r}+\frac{\partial(\rho u E)}{\partial x}= & \frac{1}{r} \frac{\partial}{\partial r}\left[r\left(v P_{r r}+u P_{r x}+\kappa \frac{\partial T}{\partial r}\right)\right] \\
& +\frac{\partial}{\partial x}\left(v P_{x r}+u P_{x x}+\kappa \frac{\partial T}{\partial x}\right)
\end{aligned}
$$

in which, $\mathrm{T}$ is the temperature and $\kappa$ is the heat transfer coefficient of the fluid.

4. Equation of state:

$$
p=\rho R T
$$


5. The basic equations of turbulent flow is as follows:

$$
\left\{\begin{aligned}
\frac{\partial}{\partial t}(\rho u)+\frac{1}{r} \frac{\partial}{\partial r}(\rho r u v)+\frac{\partial}{\partial x}(\rho u) & =-\frac{\partial p}{\partial x}+ \\
v & {\left[\frac{1}{r} \frac{\partial}{\partial \mathrm{r}^{2}}(\rho \mathrm{ru})+\frac{\partial^{2}}{\partial r^{2}}(\rho \mathrm{u})+\frac{\partial^{2}}{\partial x^{2}}(\rho \mathrm{u})\right] } \\
\frac{\partial}{\partial t}(\rho v)+\frac{1}{r} \frac{\partial}{\partial r}(\rho r v v)+\frac{\partial}{\partial r}(\rho v) & =-\frac{\partial p}{\partial r}+ \\
v & {\left[\frac{1}{r} \frac{\partial}{\partial \mathrm{r}^{2}}(\rho \mathrm{rv})+\frac{\partial^{2}}{\partial r^{2}}(\rho \mathrm{v})+\frac{\partial^{2}}{\partial x^{2}}(\rho \mathrm{v})\right] }
\end{aligned}\right.
$$

\subsection{Initial and boundary conditions}

The parameters of the computational domian is equal to the ambient at the initial. To the inlet of the computational domain, i.e, the outlet of the nozzle, the parameters are due to the experiment: $p=p(t), T=T(t)$

In the stepped-wall or the cylinder computational domain, there is reverse flow as the jet compacts to the wall for the effects of chamber structure on the gas expansion. The wall boundary is the fixed wall. The face of the chamber opposite to the nozzle is the outlet face. The outlet pressure is set equal with the atmosphere pressure before the plasma expands to the outlet face.

\section{Numerical simulation on expansion performance of plasma jet in atmosphere}

The simulated conditions is the same with the experiment condition as the discharge voltage is $2500 \mathrm{~V}$ and the nozzle diameter is $4 \mathrm{~mm}$. The expansion processes of plasma jet in atmosphere are simulated by Fluent software. The distribution characteristics of pressure, density, temperature and velocity have been got.

Figure 14 shows the distribution contours of pressure $(\mathrm{Pa})$, density $\left(\mathrm{kg} / \mathrm{m}^{3}\right)$, temperature $(\mathrm{K})$ and velocity $(\mathrm{m} / \mathrm{s})$ at the initial of the plasma jet as $\mathrm{t}=25 \mu \mathrm{s}$. As shown in the figure, there is a Mach disc like pressure peak at the plasma jet head. The pressure, density and temperature in this region are all larger. While, upward the Mach disc, the pressure, density and temperature are lower but the velocity is higher.

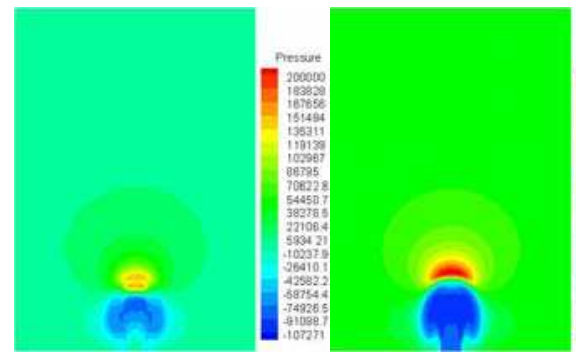

a) Relative static pressure b)Density

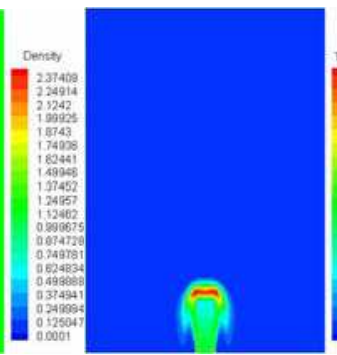

c) Temperature

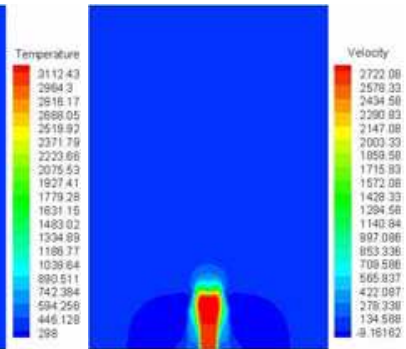

d) Velocity

Fig. 14. Contours of pressure, density, temperature and velocity at the initial of plasma jet 


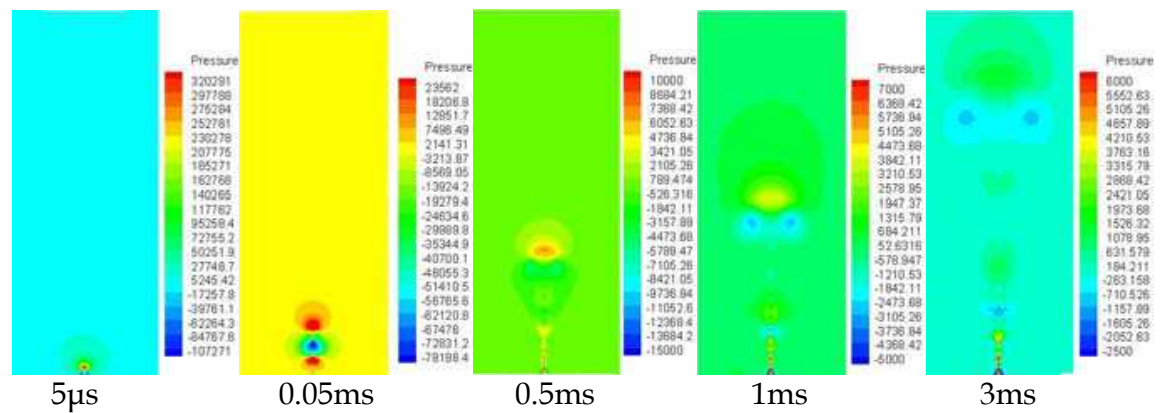

a) Relative static pressure
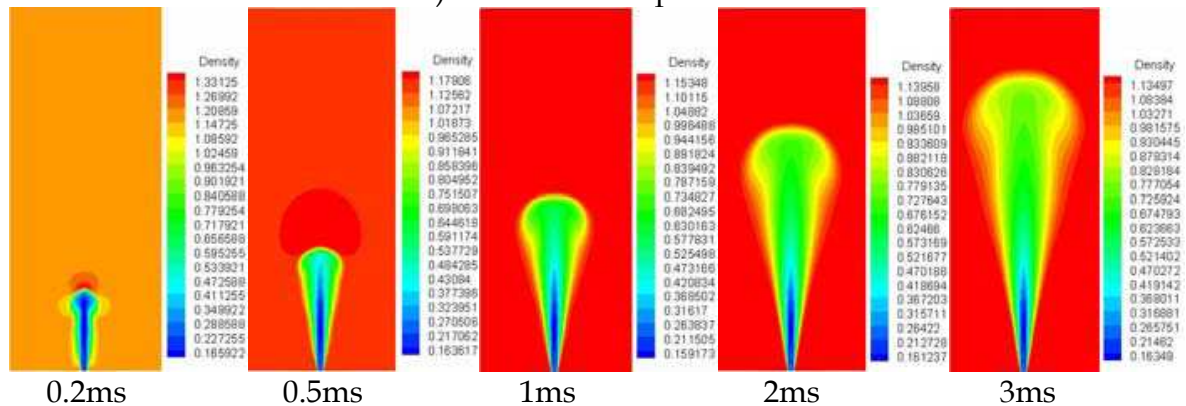

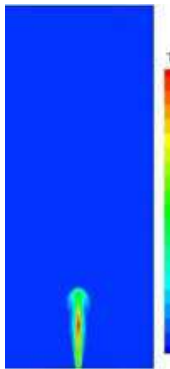

$0.2 \mathrm{~ms}$

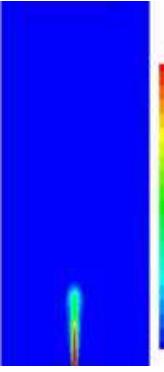

$0.2 \mathrm{~ms}$

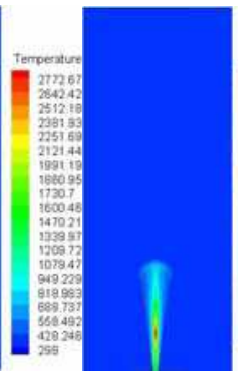

$0.5 \mathrm{~ms}$

b) Density

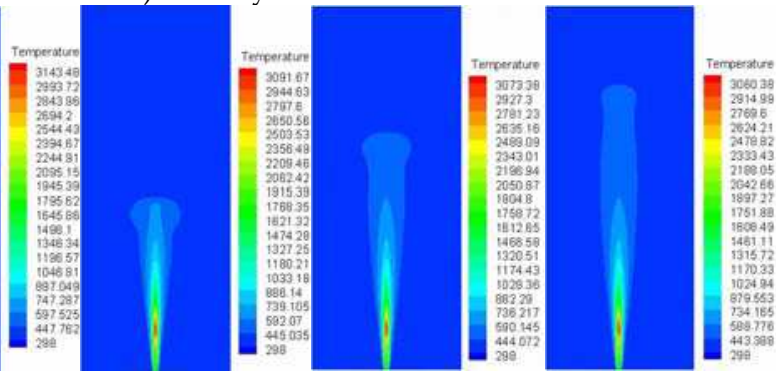

$1 \mathrm{~ms}$

$2 \mathrm{~ms}$

$3 \mathrm{~ms}$

c) Temperature

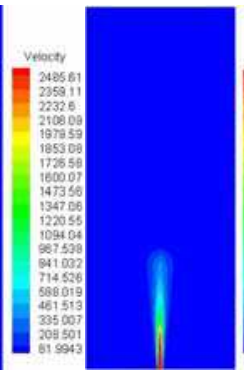

$0.5 \mathrm{~ms}$

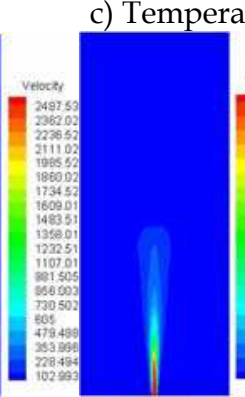

$1 \mathrm{~ms}$

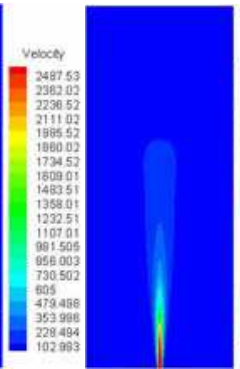

$2 \mathrm{~ms}$

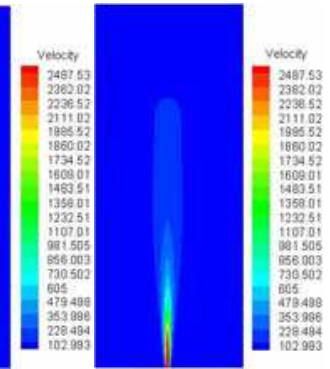

$3 \mathrm{~ms}$

d) Velocity

Fig. 15. Contours of pressure, density, temperature and velocity of the plasma jet 
Figure 15 shows the sequence contours of relative static pressure(Pa), density $\left(\mathrm{kg} / \mathrm{m}^{3}\right)$, temperature $(\mathrm{K})$ and velocity $(\mathrm{m} / \mathrm{s})$ as the plasma jet expands in the atmosphere. The pressure wave moves forward in a sphere shape as the plasma jet out the nozzle as show in figure 15(a). The pressure is alternated from high to low in the flow field during the develop processes and the pressure is fluctuated in space. The pressure fluctuation close to the nozzle is intense. The pressure of the jet head is high all along. As the time goes on, the pressure of the flow field is close to the ambient pressure.

As shown in figure $15(\mathrm{~b})$, at $\mathrm{t}=0.2 \mathrm{~ms}$, the plasma is compressed strongly for the great high pressure at the plasma jet head, and the gas density is relatively high. As the time goes on, the pressure at the plasma jet head deceases fast and the gas density is close to the ambient density gradually. As shown in figure 15 (c) the temperature increases at first and decreases then with the increases of the axial displacement away from the nozzle. The temperature along the radial direction. As shown in figure 15 (d), the velocity both decreases along the axial and radial direction.

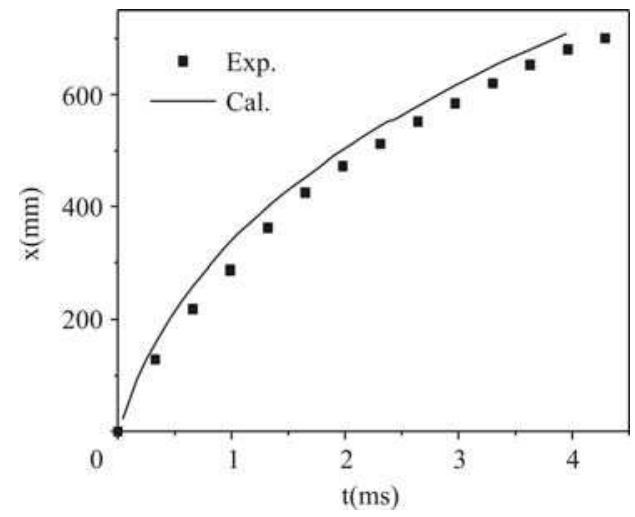

Fig. 16. Changing of the axial expansion displacement of plasma jet with time

The axial expansion displacement of plasma jet can be handled out through the sequence pictures of density. Figure 16 shows the simulated and experiment results of the axial displacement of plasma jet. They match well with each other as can be seen in the figure.

\section{Numerical simulation on expansion performance of plasma jet in liquid}

According to the experiment condition, the processes that the plasma jet into the liquid medium are simulated both in the cylinder and the cylindrical stepped-wall structures to study the parameters distribution characteristics in the flow field.

\subsection{Numerical results of the stepped-wall boundary shape}

The simulated conditions are: The capacity of the capacitors is $45 \mu \mathrm{f}$, the charging voltage is $2300 \mathrm{~V}$, and the nozzle diameter is $2.5 \mathrm{~mm}$. The first stage of the stepped-wall chamber is $14 \mathrm{~mm}$ in diameter, and $30 \mathrm{~mm}$ in length. The later three stages are all $30 \mathrm{~mm}$ in length, and every diameter is $6 \mathrm{~mm}$ larger than its former one. The liquid medium is water.

1. The pressure distribution

Figure 17 shows the isobars of the plasma jet flow field in stepped-wall chamber, the vertical ordinate is pressure and the unit is $\mathrm{Pa}$. 


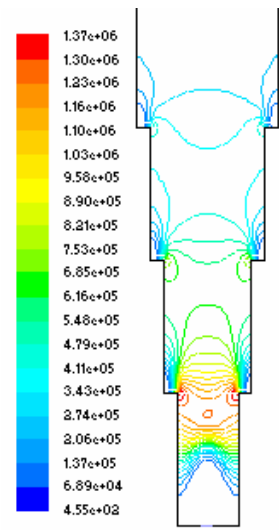

$\mathrm{t}=0.5 \mathrm{~ms}$

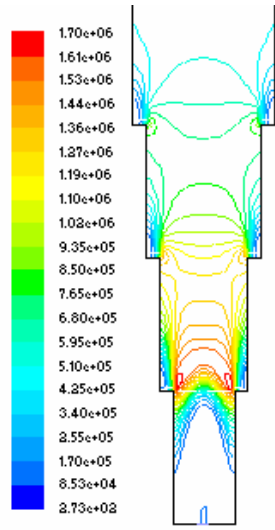

$\mathrm{t}=1 \mathrm{~ms}$

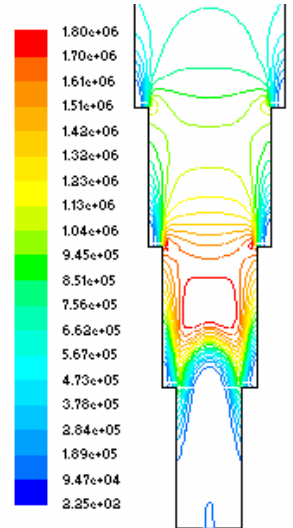

$\mathrm{t}=1.5 \mathrm{~ms}$

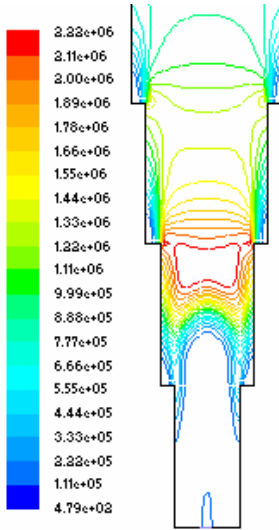

$\mathrm{t}=2 \mathrm{~ms}$

Fig. 17. Isobars of the plasma jet in stepped-wall chamber

As shown in the figure, the isobars are dense and pressure gradient is higher in the front of the plasma jet. In the initial expansion of the plasma jet, there is round pressure centre in the front of the plasma jet head. The high pressure zone grows as time goes on. At $t=1.5 \mathrm{~ms}$, the shape of the high pressure zone centre becomes cone frustum. At $t=2 \mathrm{~ms}$, the edge of the high pressure zone similar to an inverted cone frustum has a radial expansion at the 2nd step attributed to the radial induced. There is an obviously pressure fluctuation during the processes of the plasma jet expansion. When jet impinges against the wall at the steps, the reverse flow occurs, so the low pressure zones can be observed from the figure.

In order to quantitative describe the pressure distribution of the jet flow field, take the pressure at the centre axis and the section at the position $45 \mathrm{~mm}$ away from the nozzle into account. Figure 18 indicates the changes of the pressure through time at different points on the axis (the direction of jet centre axis is y longitudinal axis, perpendicular to the nozzle is $\mathrm{x}$ transverse axis).

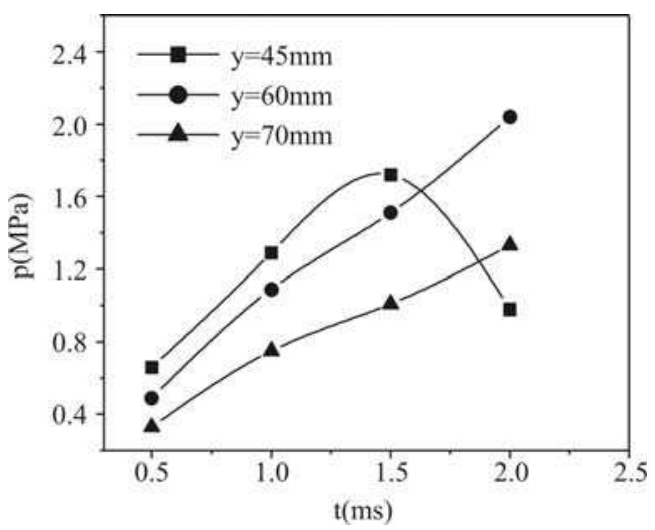

Fig. 18. Changing of the axial pressure with time

Figure 19 shows the pressure-time curves at the section which is $45 \mathrm{~mm}$ distance from the nozzle. Overall, the pressure on axis is increasing as time goes on. At the distance of $70 \mathrm{~mm}$ 
from the nozzle, the pressure has a stable increase for the further distance from the nozzle. At $y=45 \mathrm{~mm}$, the pressure has a rapid increase as time goes on. When $t=0.5 \mathrm{~ms}, \mathrm{p}=0.66 \mathrm{MPa}$. While $\mathrm{t}=1 \mathrm{~ms}, \mathrm{p}=1.29 \mathrm{MPa}$. The pressure gets to the biggest $1.72 \mathrm{MPa}$ at $\mathrm{t}=1.5 \mathrm{~ms}$, the high pressure zone propagates to $y=45 \mathrm{~mm}$ at the same time, then it goes ahead. The pressure at the surface which is $45 \mathrm{~mm}$ from the nozzle is decreasing. On the radial direction, at the section $y=45 \mathrm{~mm}$, the pressure is increasing before $t=1.5 \mathrm{~ms}$ because of the high pressure zone propagation. But the closer to the boundary, the smaller the pressure is. After $t=1.5 \mathrm{~ms}$, the high pressure zone passes across the section at $y=45 \mathrm{~mm}$ and the boundary pressure has a rapid decrease. The low pressure zone forms on the boundary.

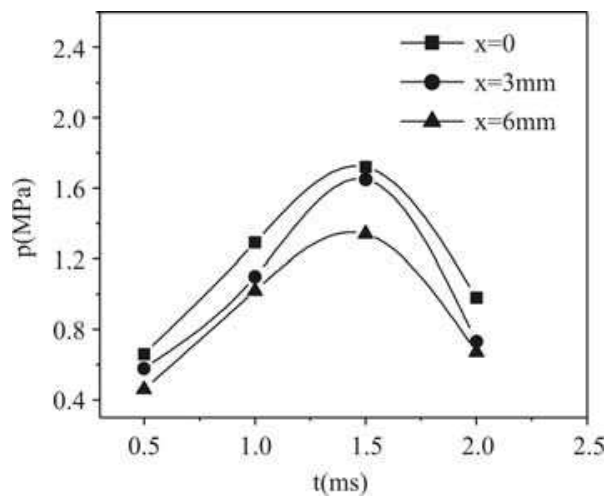

Fig. 19. Radial pressure-time curves

2. The velocity distribution

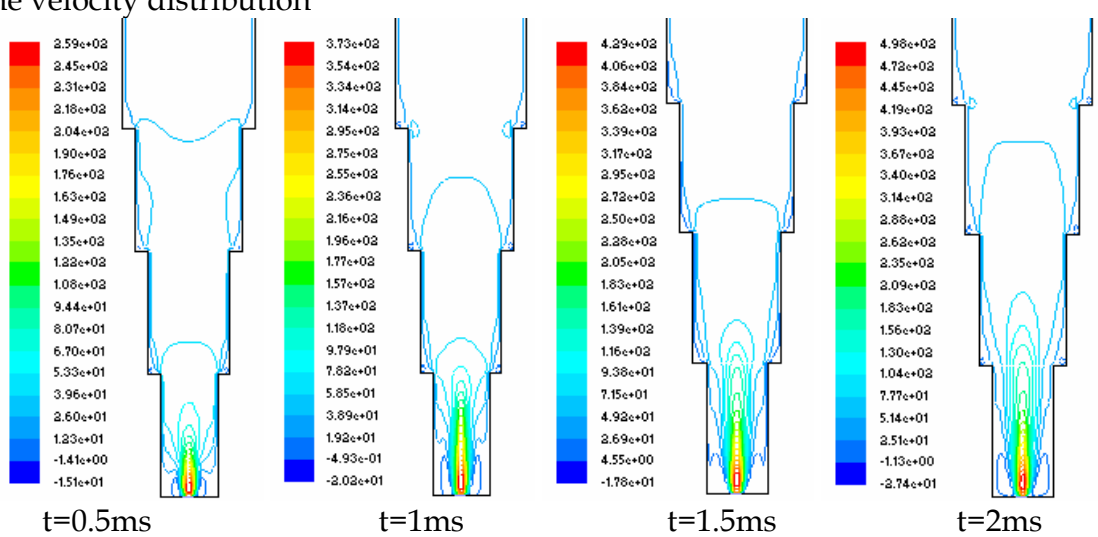

Fig. 20. Isovels of the plasma jet in stepped-wall chamber

The isovels distributions of the plasma jet expansion in the liquid are shown in figure 20 (vertical ordinate is velocity, unit: $\mathrm{m} / \mathrm{s}$ ). It can be observed from the figure that the biggest jet velocity is near the nozzle. The velocity gradient at the interface of gas and liquid is larger, in addition, the velocity is easy to decrease when the plasma jet expands in the liquid for its light quality. As shown in the figure that the velocity is very high in the jet centre but it has a sharp fall near the wall. As the time goes on, the Taylor cavity is expanding along the axial direction. At $\mathrm{t}=1.5 \mathrm{~ms}$, the head of plasma jet has crossed the first step. 


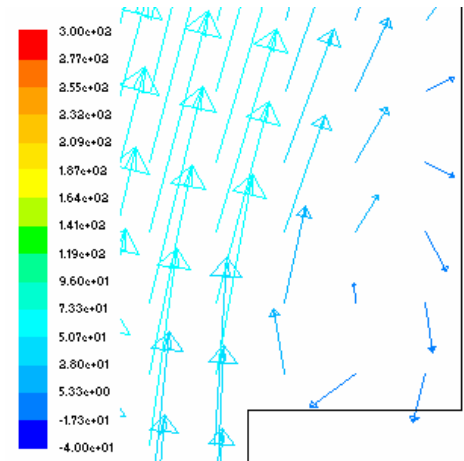

a) The corner of the step

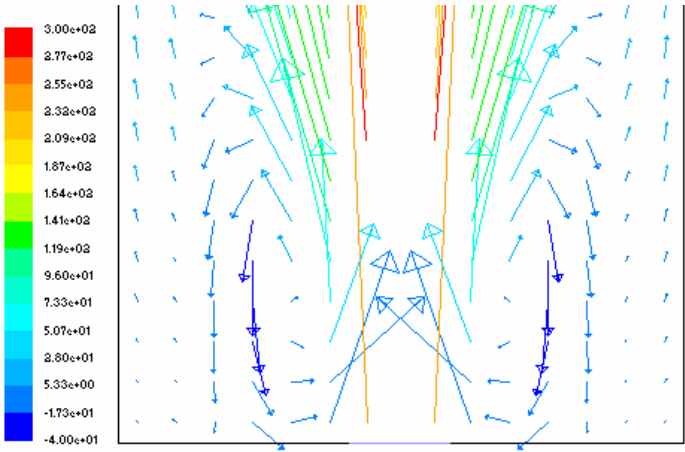

b) nozzle

Fig. 21. Partial velocity vector diagrams at the corner of the step and the nozzle

Figure 21(a) shows the velocity vector at the steps. The ring isovels and the negative velocity can be observed from the figure due to the radial turbulence and the reverse flow attributed to the impinging of the jet against the wall at steps. At $t=2 \mathrm{~ms}$, the jet head has propagated to the 1.5th step. Figure 21(b) shows the partial velocity vector at the nozzle. During the processes of the plasma jet propagation, ring isovels can be observed near the nozzle because of the strong turbulence mixture of the gas and liquid, that is the reverse flow phenomenon, and there are negative velocity can be seen in the isovels.

3. The temperature distribution

Fig.22 shows the isotherm of the plasma jet during the expansion in the stepped-wall chamber, the vertical ordinate is temperature and the unit is $\mathrm{K}$. As shown in the figure, the temperature in axial direction is higher than that in the radial direction. The temperature close to the nozzle is highest, and it reduces to the ordinary temperature in very short distance along the axis. In radial direction, the temperature also decreases readily near the nozzle due to the completely mixture of the plasma jet and the liquid. After all, plasma jet in the liquid attenuates quickly and the heat is easy to diffuse. As the expansion of the jet, the temperature reduces quickly.

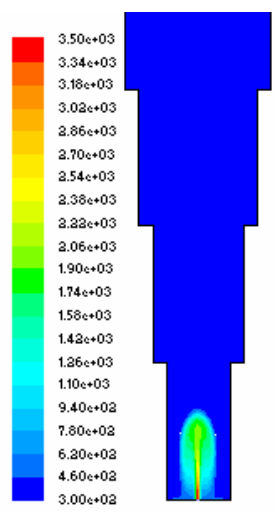

$\mathrm{t}=0.5 \mathrm{~ms}$

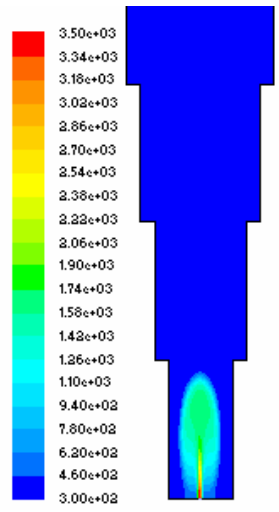

$\mathrm{t}=1 \mathrm{~ms}$

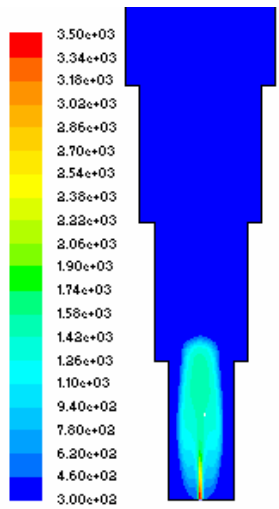

$\mathrm{t}=1.5 \mathrm{~ms}$

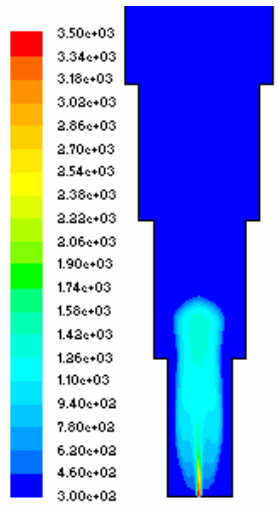

$\mathrm{t}=2 \mathrm{~ms}$

Fig. 22. Isotherms of plasma jet in the liquid 
In order to describe the temperature distribution of the jet flow field quantitative, take the value at the centre axis and the section $15 \mathrm{~mm}$ away from the nozzle into account. Figure 23 indicates the changes of the temperature through time at different points $10 \mathrm{~mm}, 15 \mathrm{~mm}$ and $25 \mathrm{~mm}$ away from the nozzle on jet centre axis. Figure 24 shows the temperature-time curve at the section which is $15 \mathrm{~mm}$ distance from the nozzle.

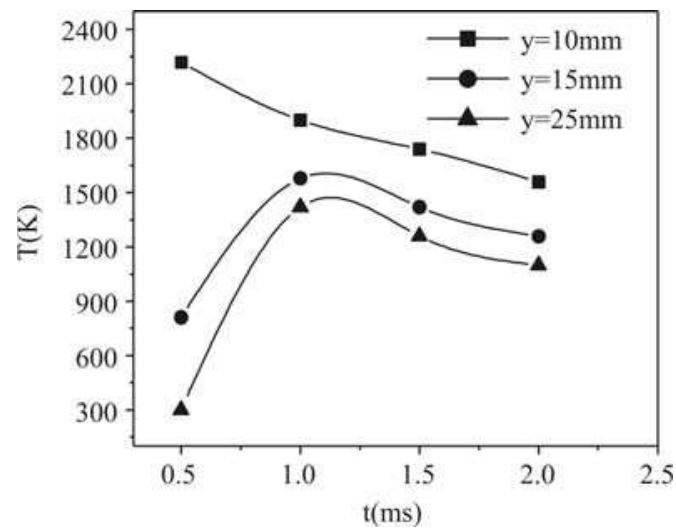

Fig. 23. Changing of axial temperature with time

As shown in the figure, the axial temperature is higher at the position nearer to the nozzle. At $\mathrm{t}=0.5 \mathrm{~ms}$, the temperature is $2300 \mathrm{~K}$ at the position $10 \mathrm{~mm}$ away from the nozzle, $812 \mathrm{~K}$ at $15 \mathrm{~mm}$ away from the nozzle and $300 \mathrm{~K}$ at $25 \mathrm{~mm}$ away from the nozzle; as time goes on, the temperature increases gradually at $15 \mathrm{~mm}$ and $25 \mathrm{~mm}$ away from the nozzle. At $t \approx 1 \mathrm{~ms}$, the temperature gets the largest value and then decreases. And the temperature are $1600 \mathrm{~K}$, $1260 \mathrm{~K}$ and $1100 \mathrm{~K}$ respectively at the three point $(10 \mathrm{~mm}, 15 \mathrm{~mm}$ and $25 \mathrm{~mm}$ away from the nozzle); at the section of $15 \mathrm{~mm}$ away from the nozzle, the radial temperature decreases faster, the temperature is lower at the position nearer the boundary, at $t=2 \mathrm{~ms}$ the temperature is $1260 \mathrm{~K}, 900 \mathrm{~K}$ and $760 \mathrm{~K}$ at the radial position of $0 \mathrm{~mm}, 3 \mathrm{~mm}$ and $6 \mathrm{~mm}$ of the section $15 \mathrm{~mm}$ away from the nozzle respectively.

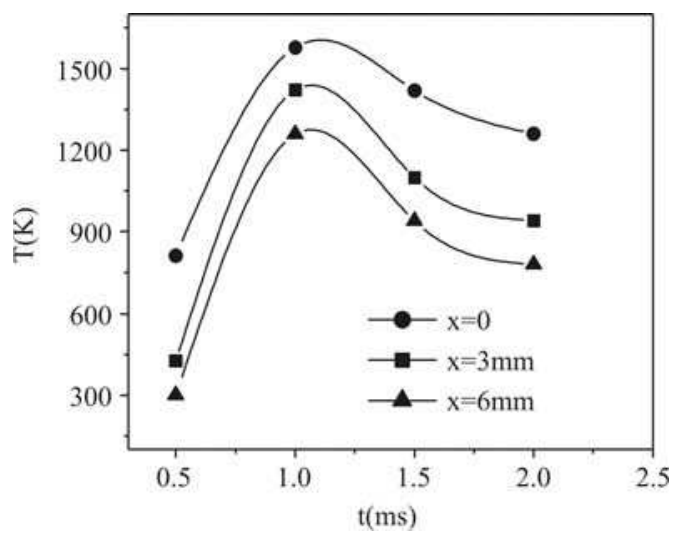

Fig. 24. Changing of radial temperature with time 
Through the isothermal, the Taylor cavity expansion displacement of plasma jet at different time can be got. Figure 25 shows the compare of the simulated value with the experimental results shown in figure 12(b). As shown in the figure, they mach well with each other.

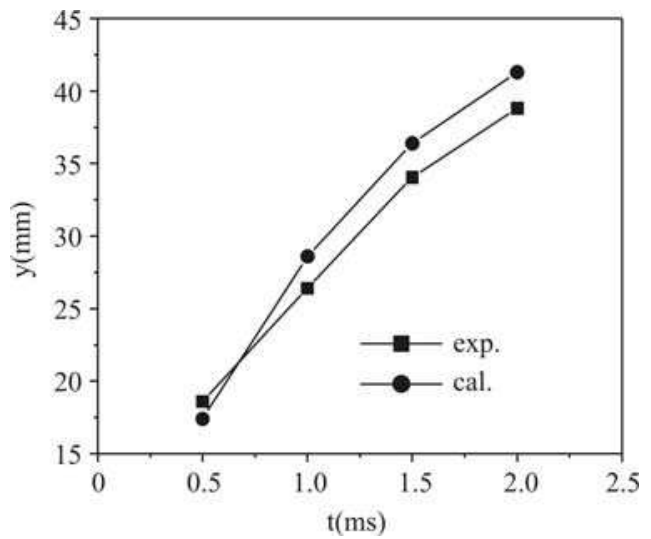

Fig. 25. Compare of the experiment and the calculated value of the Taylor cavity

\subsection{Numerical results of the cylindrical boundary shape}

The simulated conditions are as follows: The capacity of the capacitors is $45 \mu \mathrm{f}$, the charging voltage is $2300 \mathrm{~V}$, and the nozzle diameter is $2.5 \mathrm{~mm}$. The diameter of the cylindrical chamber is $26 \mathrm{~mm}$ and its total length is $107 \mathrm{~mm}$. The liquid medium is water.

1. The pressure distribution

Figure 26 shows the isobars of the plasma jet in cylinder chamber, the vertical ordinate is pressure and the unit is $\mathrm{Pa}$. The pressure gradient is higher and the isobars are dense on the interface of the plasma jet and the liquid. There is a larger high pressure region in a tapered shape in front of the jet head. It grows and moves forward gradually. The expansion velocity in axial direction is larger than that in radial direction and the low pressure region forms at the boundary of the chamber which can be seen in the figure.
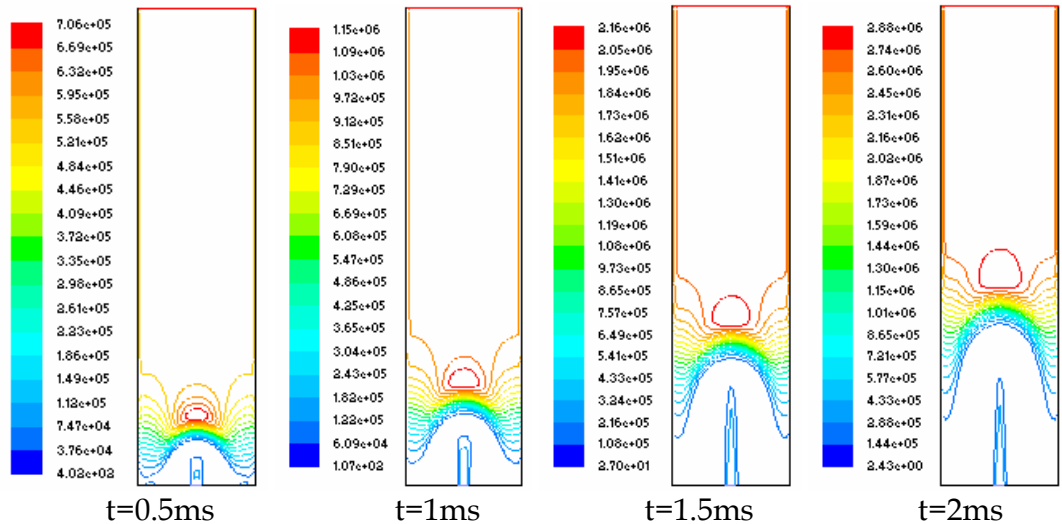

Fig. 26. Isobars of the plasma jet in liquid in cylinder chamber 
Compared with the stepped-wall structure, the isobars distribution are different, especially the high pressure region's shape. The high pressure region moves keeping a tapered shape in the cylindrical chamber. While in the stepped-wall chamber, the high pressure region is in a cone frustum shape, the high pressure region expands along the radial direction and there is low pressure region both at the boundary and the steps due to the entrainment of the stepped-wall shape.

2. The velocity distribution

The isovels distributions of the plasma jet expansion in the liquid in the cylindrical chamber are shown in figure 27 (vertical ordinate is velocity, unit: $\mathrm{m} / \mathrm{s}$ ).

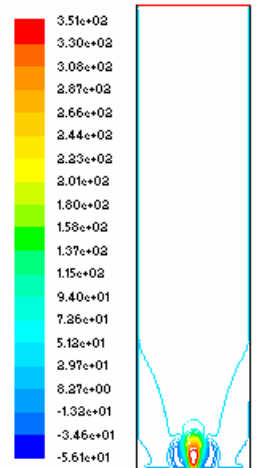

$\mathrm{t}=0.5 \mathrm{~ms}$

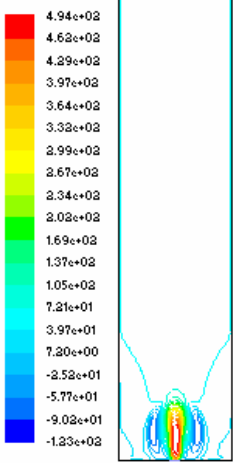

$\mathrm{t}=1 \mathrm{~ms}$

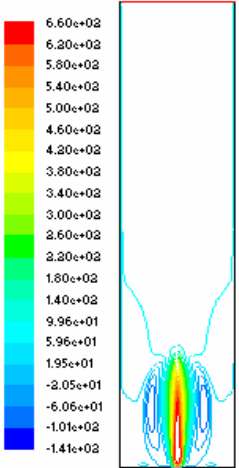

$\mathrm{t}=1.5 \mathrm{~ms}$

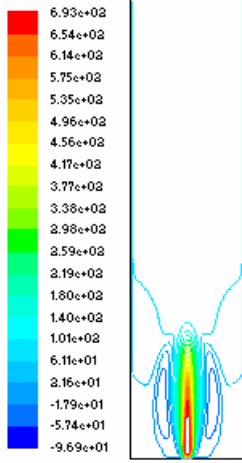

$\mathrm{t}=2 \mathrm{~ms}$

Fig. 27. Isovels of the plasma in the liquid in cylinder chamber

In the cylindrical chamber, the isovels are dense near the nozzle and the largest velocity is on the axis. The reverse flow forms around the largest velocity region. The reverse flow region grows during the expansion. And there is great disturbance on the gas-liquid interface.
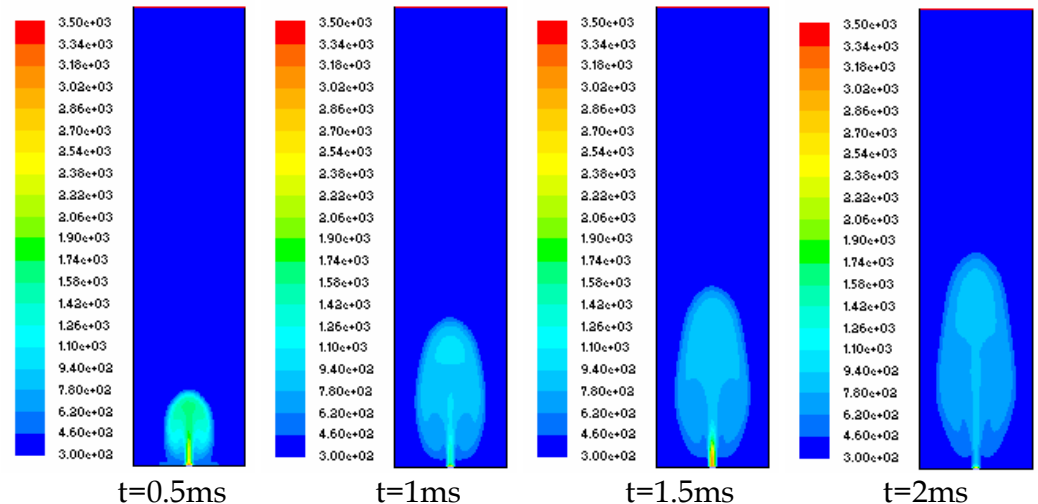

Fig. 28. Isotherms of the plasma jet in the liquid in cylinder chamber

Compared with the stepped-wall chamber, the isovels are denser near the axis and the velocity gradient is bigger on the gas-liquid interface. Due to the radial induction of the 
steps in the stepped-wall chamber, the jet momentum diffuses along the radial direction of steps. The isovels' gradient of the jet head is lower at the steps.

3. The temperature distribution

Figure 28 shows the isotherms of the plasma jet during the expansion in the stepped-wall chamber, the vertical ordinate is temperature and the unit is $\mathrm{K}$. As can be seen in the figure, in the cylindrical chamber, the axial expansion of the jet is obvious and the radial expansion is slower relatively. There is a tapered isothermal region at the head of the jet and it moves forward. The temperature at the nozzle is highest and it reduces quickly along the axial direction. Compared with the stepped-wall chamber, the temperature decreases more easily along the axial direction in the cylinder chamber.

According to the isotherm, the expansion displacement of the Taylor cavity can be acquired. Figure 29 shows the comparisons between the numerical simulation results and the experimental results. They coincide well with each other.

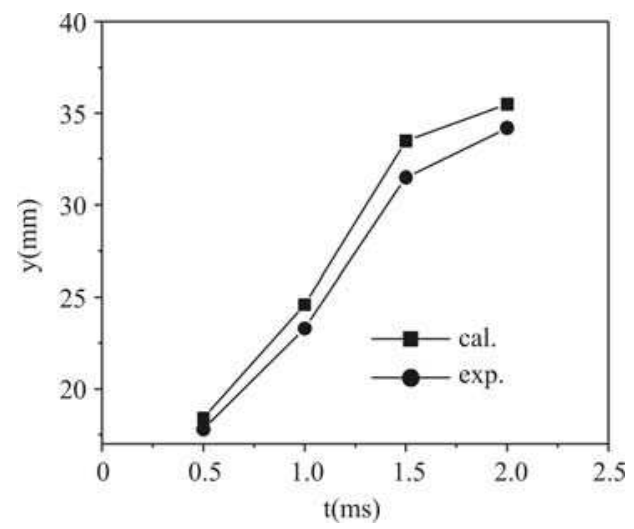

Fig. 29. Compare of the experiment and the calculated value of the Taylor cavity's displacement

\subsection{Numerical results of different discharge voltages}

According the experimental condition as show in figure 12, the stepped-wall structure is: the nozzle diameter is $2.5 \mathrm{~mm}$, the capacity of the capacitor group is $45 \mu \mathrm{F}$, and the discharge voltage is $2000 \mathrm{~V}, 2300 \mathrm{~V}$ and $2500 \mathrm{~V}$ respectively. And the discharge jet energy is 36J, 48J and 56J respectively taking the conversion efficiency of the pulse electrical source is about $40 \%$ into account. At these conditions, the effects of different discharge voltage on the plasma jet are simulated.

1. The pressure distribution

Figure 30 shows the isobars of the plasma jet on different discharge voltage, the vertical ordinate is pressure and the unit is $\mathrm{Pa}$. As shown in the figure, the larger is the discharge voltage, the earlier the pressure centre of the jet head forms in a cone frustum shape, and the pressure value at the centre is larger. The discharge voltage in figure 30 (a) is the least and there is no obvious pressure centre in $2 \mathrm{~ms}$. In figure $30(\mathrm{~b})$, there is a cone frustum pressure centre at $\mathrm{t}=1.5 \mathrm{~ms}$ and the high pressure value is $1.80 \mathrm{MPa}$. While in figure 30 (c), the cone frustum pressure centre forms as $t=1 \mathrm{~ms}$ and the high pressure value is $2.05 \mathrm{MPa}$. Otherwise, in figure 30 (c), due to the radial expansion, the cone frustum pressure centre is stretched, there are two small pressure centres at the $2^{\text {nd }}$ step and move according the boundary of step. 


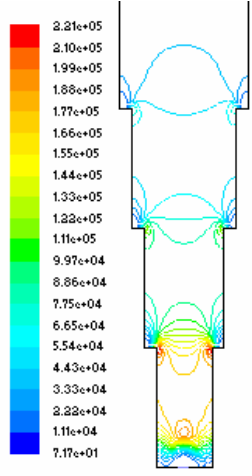

$\mathrm{t}=0.5 \mathrm{~ms}$

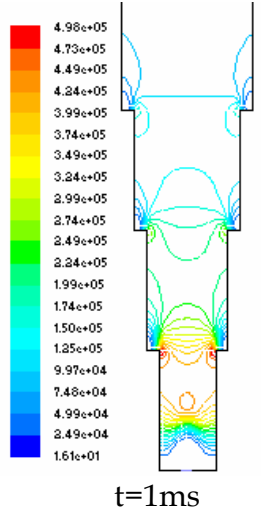

$\mathrm{t}=1 \mathrm{~ms}$

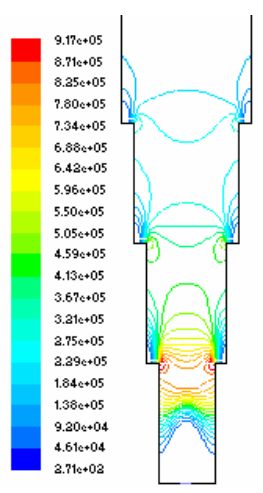

$\mathrm{t}=1.5 \mathrm{~ms}$

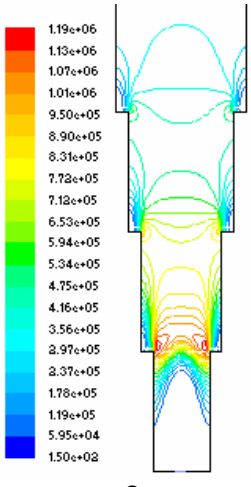

$\mathrm{t}=2 \mathrm{~ms}$

a) Discharge voltage is $2000 \mathrm{~V}$
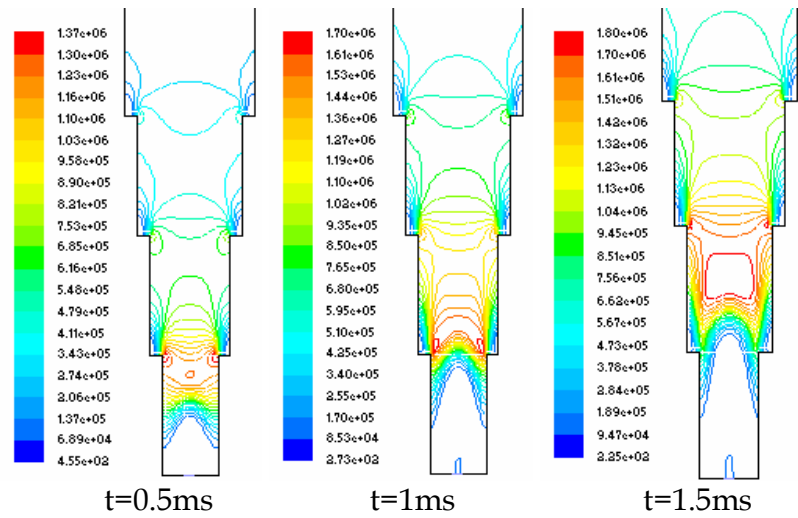

$\mathrm{t}=1.5 \mathrm{~ms}$

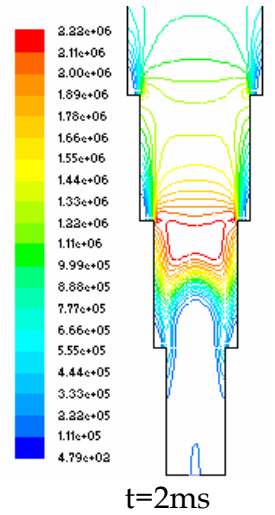

b) Discharge voltage is $2300 \mathrm{~V}$
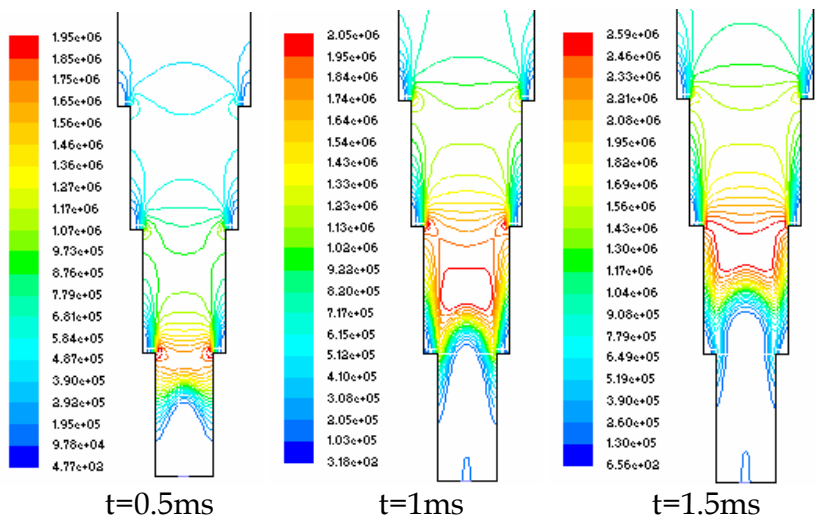

$\mathrm{t}=1.5 \mathrm{~ms}$

c) Discharge voltage is $2500 \mathrm{~V}$

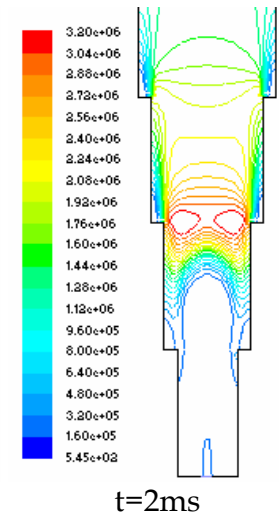

$\mathrm{t}=2 \mathrm{~ms}$

Fig. 30. Isobars of the plasma jet at different discharge voltages 
2. The velocity distribution
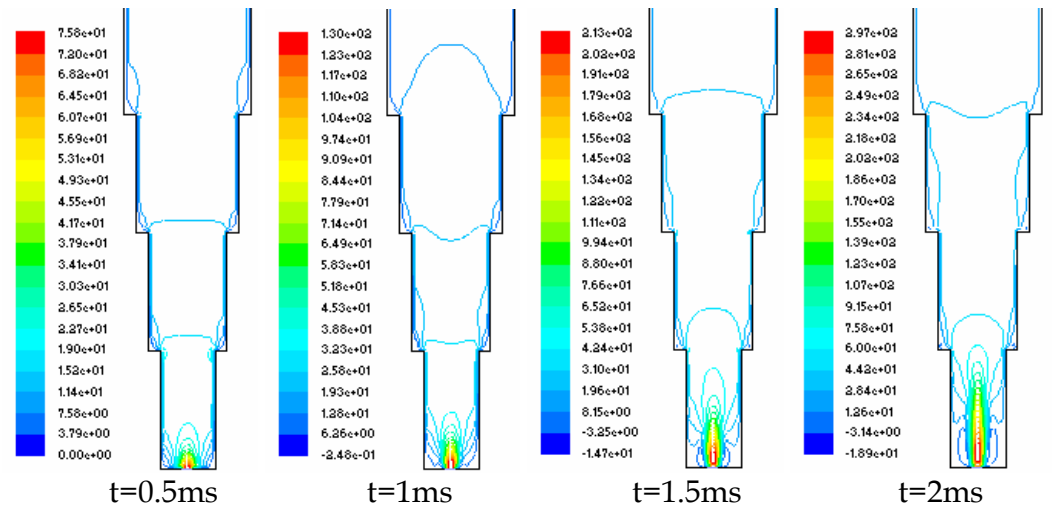

a) Discharge voltage is $2000 \mathrm{~V}$

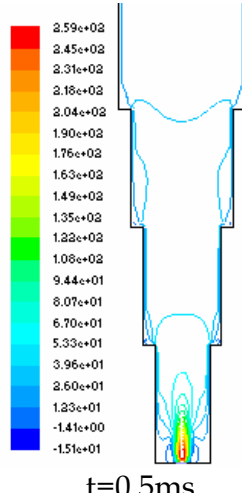

$\mathrm{t}=0.5 \mathrm{~ms}$

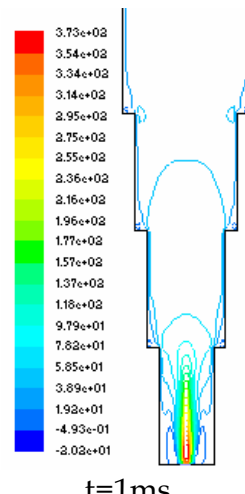

$\mathrm{t}=1 \mathrm{~ms}$

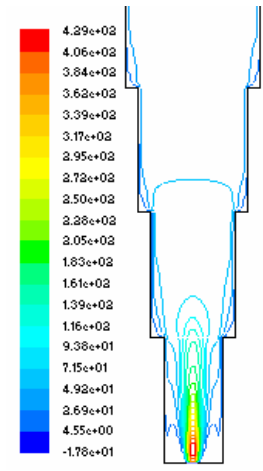

$\mathrm{t}=1.5 \mathrm{~ms}$

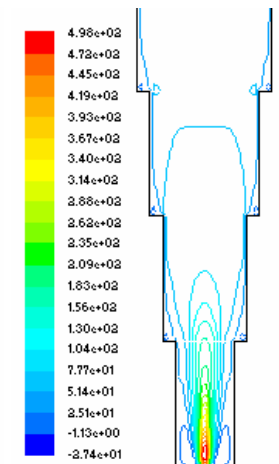

$\mathrm{t}=2 \mathrm{~ms}$

b) Discharge voltage is $2300 \mathrm{~V}$

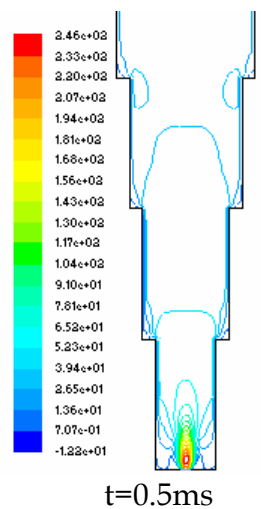

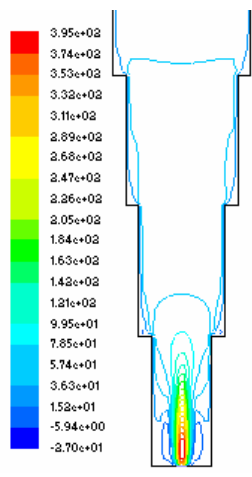

$\mathrm{t}=1 \mathrm{~ms}$

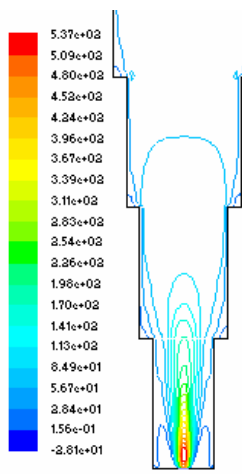

$\mathrm{t}=1.5 \mathrm{~ms}$

c) Discharge voltage is $2500 \mathrm{~V}$

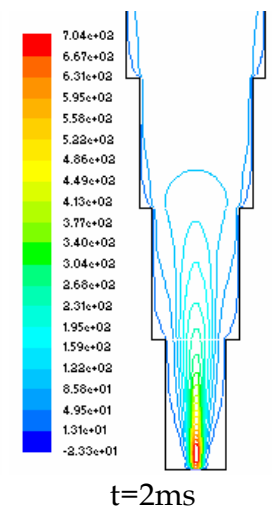

$\mathrm{t}=2 \mathrm{~ms}$

Fig. 31. Isovels of the plasma jet at different discharge voltages 


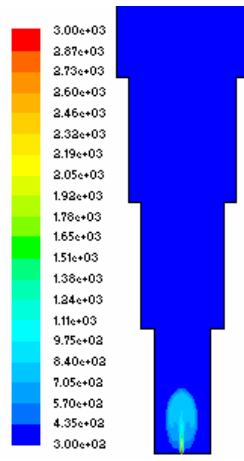

$\mathrm{t}=0.5 \mathrm{~ms}$

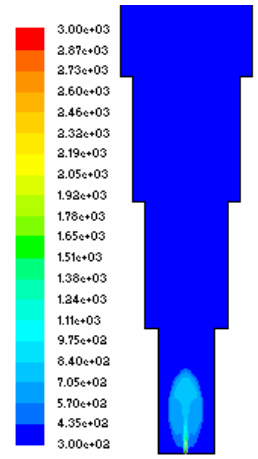

$\mathrm{t}=1 \mathrm{~ms}$

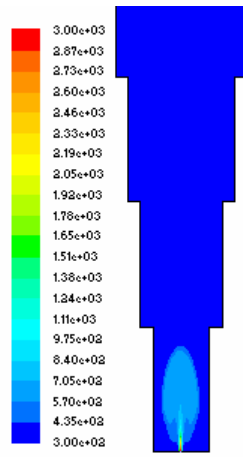

$\mathrm{t}=1.5 \mathrm{~ms}$

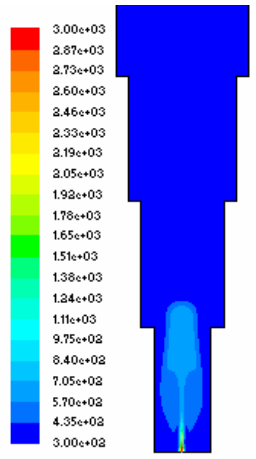

$\mathrm{t}=2 \mathrm{~ms}$

a) Discharge voltage is $2000 \mathrm{~V}$
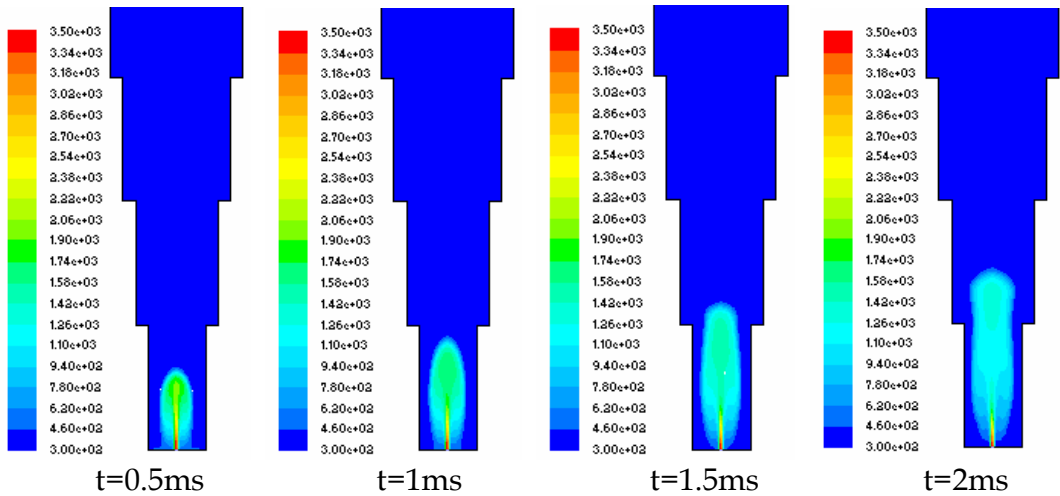

b) Discharge voltage is $2300 \mathrm{~V}$
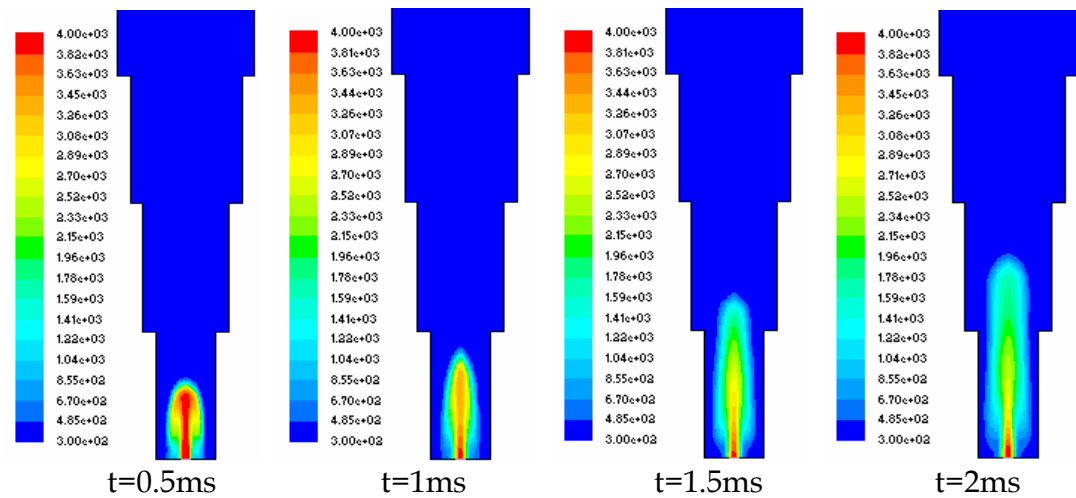

c) Discharge voltage is $2500 \mathrm{~V}$

Fig. 32. Isotherms of the plasma jet at different discharge voltages 
Figure 31 shows the isovels of the plasma jet, the vertical ordinate is velocity and the unit is $\mathrm{m} / \mathrm{s}$. As shown in the figure, the larger is the discharge voltage, the expansion of the region which has the biggest jet velocity is faster. At $t=1.5 \mathrm{~ms}$, the biggest velocity region expand to $15 \mathrm{~mm}$ away from the nozzle in figure 31 (a), $30 \mathrm{~mm}$ away from the nozzle in figure 31 (b) and about $35 \mathrm{~mm}$ away from the nozzle in figure 31 (c). The larger is the discharge voltage, the isovels are denser near the jet core, the velocity gradient is larger on the gas-liquid interface, the bottom reverse flow region forms earlier and attenuation is also faster. In figure 31(c), there are no obvious reverse flow isovels at $\mathrm{t}=2 \mathrm{~ms}$.

3. The temperature distribution

Figure 32 shows the isotherms of the plasma jet at different discharge voltages, the vertical voltage is temperature and the unit is $\mathrm{K}$. As shows in the figure, the larger is the discharge voltage, the higher is the temperature at the nozzle and the temperature increases faster, the high temperature region in figure 32(c) is more obvious; the larger is the discharge voltage, the slower is the heat dissipation of the jet.

Take the temperature change at the position $20 \mathrm{~mm}$ away from the nozzle for example to illustrate the effect of the discharge on the temperature. At $t=1.5 \mathrm{~ms}$, in figure $32(\mathrm{a})$, the temperature is $970 \mathrm{~K}$, the temperature is $1530 \mathrm{~K}$ in figure $32(\mathrm{~b})$ at the same time which is 1.6 times to the value in figure 32 (a), and the temperature is $3320 \mathrm{~K}$ in figure $32(\mathrm{c})$ at $\mathrm{t}=1.5 \mathrm{~ms}$ which is 3.3 times to the value in figure 32 (a).

\section{Conclusions}

The experiment and the theoretical study of the expansion characteristics of the plasma jet both in atmosphere and the bulk-loaded liquid medium are mainly discussed in this chapter. The expansion processes of the plasma jet are recorded by the high speed camera system, and the effects of the discharge energy and the chamber structures on the plasma jet expansion processes are analysesed. Two-dimensional axial symmetry model of the interaction between the plasma jet and the liquid medium are proposed based on the experiment and the simulations are conducted. The change characteristics of pressure, temperature and velocity in the jet flowfield are got. According to the experiment and the simulation results, the following conclusions can be got:

1. During the expansion of the plasma jet in atmosphere, the shape of jet head changes from ellipsoid to taper as the going of the expansion. The brightness of the jet enhances at first then decays. The jet head is brightest. The axial and the radial expansion velocity both have a fluctuation and the axial velocity is larger than the radial one. The later peak is lower than the former one which can be seen from the distribution of the axial velocity changing with time.

2. As soon as the plasma eject into the atmosphere, there is a sphericity pressure wave at the nozzle exit. As the going of the expansion, the pressure wave moves and attenuates quickly. The pressure alternates from high to low at the initial expansion stage.

3. There is intense turbulence dissipation during the expansion of the plasma jet in atmosphere. The jet head is in drape shape at first and the turbulence is strengthened as the gonging of the expansion, the turbulence mixture region grows. The larger is the discharge voltage, the greater is the plasma jet initial expansion velocity, and the reverse flow entrainment and dissipation are more intense. While the relationship between the axial displacement of the plasma jet and the discharge voltage is not monotony and there is a critical discharge voltage. 
4. As the plasma jet into the liquid, the initial expansion velocity of the Taylor cavity is higher. As the Taylor cavity moves downwards, the velocity decreases and the axial velocity is larger than the radial one. The lightness of the jet head decays as the jet develops to some stage which is caused by the water vapor and the temperature decreases. There is intense heat and mass transfer between the plasma and liquid on the Taylor cavity surface.

5. The structure of the inspection chamber affects the shape and expansion velocity of Taylor cavity. The radial disturbance of the boundary structure to the plasma jet in stepped-wall chamber is higher than that in the cylinder chamber. There is subsection phenomenon during the plasma expansion and the lower is the plasma jet energy, the earlier is the subsection shows which cannot be seen in the cylinder structure.

6. The high pressure region of plasma jet head moves keeping the taper shape in the cylinder chamber; while in stepped-wall chamber, the high pressure is in cone frustum shape at initial, and the high pressure expands along the radial due to the radial entrainment of the steps, there are two small pressure center and moves towards the steps. The larger is the discharge voltage, the higher is the kinetic pressure at the jet axis and the pressure gradient is bigger.

7. The isovels are dense near the nozzle and the jet core, the velocity gradient is larger on the interface. The further away from the nozzle, the smaller is the velocity. The velocity at the axis is highest. There is reverse flow near the jet core which has the biggest velocity and the reverse flow region grows as time goes on. There is also reverse flow at the step corner in the stepped-wall structure, and the minus velocity occurs.

8. In cylinder chamber, the isotherms of plasma jet head moves keeping in taper shape while in blunt body shape in the stepped-wall chamber. The axial temperature is higher than the radial one. The temperature decreases rapidly as the going of the jet. The larger is the discharge voltage, the higher is the temperature near the nozzle and the temperature at the axis increases faster.

\section{Acknowledgement}

This work is supported by National Nature Science Foundation of China (No.50776048).

\section{References}

Arensburg, A. (1993). X-ray diagnostics of a plasma-jet-liquid interaction in electrothermal guns. Journal of Applied Physics, Vol. 5, No. 73, (1993), pp. 2145-2154, ISSN 0021-8979

Chang, L M. \& Howard, S L. (2007). Influence of Pulse Length on Electrothermal Plasma Jet Impingement Flow. ARL-TR-4348

Guo, H B.; Liu, D Y. \& Zhou, Y H. (2007). Experimental Study of Pulsed Discharge Property of Plasma Generator. Journal of Nanjing University of Science and Technology(Natural Science), Vol. 4, No. 31, (2007), pp. 466-469, ISSN 1005-9830

Hsioa, C.; Phillips, G. \& Su, F. (1993). A Numerical Model for ETC Gun Interior Ballistics Applications. IEEE Transaction on Magnetics, Vol. 1, No. 29, (1993), pp. 567-572, ISSN 0018-9464

Kim, H J.\& Hong, S H. (1995). Comparative Measurements on Thermal Plasma Jet Characteristics in Atmospheric and Low Pressure Plasma Sprayings. IEEE Transactions on Plasma Science, Vol. 5, No. 23, (1995), pp. 852-858, ISSN 0018-9464 
Kim, J U. \& Suk, H. (2002). Characterization of High-density Plasma Produced by Elecrtothermal Capillary Discharge. Applied Physics Letters, Vol. 3, No. 80, (2002), pp. 368370, ISSN 0003-6951

Kuo K K., Cheung, F B., \& Hsieh, W H. (1990).Experiments study of plasma/fluid interaction in a simulated CAP gun, 27th JANNAF combustion subcommittee meeting, pp.365-375, Maryland, USA, 1990(1)

Li, X Q. ; Hong, Y J. \& He, G Q. (2010). Reviews of the Propulsive Characteristics Study on Liquid Propellants for Laser Propulsion. Journal of Propulsion Technology, Vol. 1, No. 31, (2010), pp. 105-110, ISSN 1001-4055

Robert, H F.(2003). Advanced Space Propulsion for the 21st Century. Journal of Propulsion and Power, Vol. 6, No. 9, (2003) , pp.1129-1154, ISSN 0748-4568

Rott M. , Artelt C. \& Höschen T. (2005). Experimental Investigation of Hypervelocity Plasma Jets Generated with a Coaxial Arc Device. IEEE Transactions on Magnetics, Vol. 1, No. 41, (2005), pp. 220-225, ISSN 0018-9464

Taylor, M J. (2001). Measurement of the Properties of Plasma from ETC Capillary Plasma Generators. IEEE Transactions on Magnetics, Vol. 1, No. 37, (2001), pp. 194-198, ISSN 0018-9464

Weidong, S. (2004). Active Electrospray Ionization for Efficient Electric Thrusters. AIAA 2004-3942, 2004

Wilson, D E. \& Kim, K J. (1999). Theoretical Analysis of an External Pulsed Plasma Jet. IEEE Transaction on Magnetics, Vol. 1, No. 35, (1999), pp. 228-233, ISSN 0018-9464

Yu, Y G. , Yan S H. \& Zhao N. (2009) Influence of Boundary Shape on Interaction Process of Plasma Jet and Liquid Media, Proceedings of the 14th International Symposium on Applied Electromagnetics and Mechanics, pp. 197-198, Xian, China, 2009.9, ISBN 978-4931455-14-6

Yu, Y G. ; Yan, S H. ; Zhao, N. et al. (2009). Experimental Study and Numerical Simulation on Interaction of Plasma Jet and Liquid media, 2009 Asia-Pacific Power and Energy Engineering Conference, pp. 3750-3754, Wuhan,China,2009.3,ISBN 978-1-4244-2487-0

Zhang, Q. ; Yu, Y G. ; Lu, X. et al. (2009). Study on Propagation Properties of Plasma Jet in Atmosphere, Proceedings of the 2009 International Autumn Seminar on Propellants, Explosives and Pyrotechnics, pp. 463 468, Kunming, China,2009.9, ISBN 978-7-03025394-1

Zhou, Y H. ; Liu D Y. \& Yu Y G. (2003). Expansion characteristics of transient plasma jet in liquid. Journal of Nanjing University of Science and Technology (Natural Science), Vol. 5, No. 27, (2003), pp. 525-529, ISSN 1005-9830 


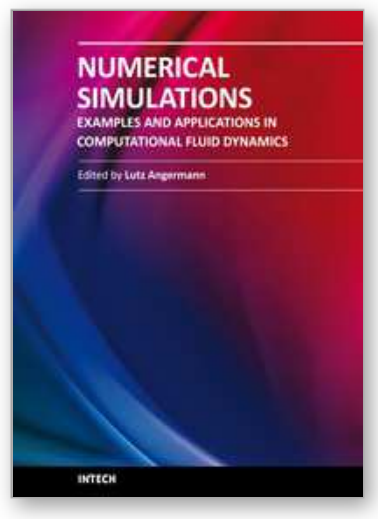

\section{Numerical Simulations - Examples and Applications in Computational Fluid Dynamics}

Edited by Prof. Lutz Angermann

ISBN 978-953-307-153-4

Hard cover, 440 pages

Publisher InTech

Published online 30, November, 2010

Published in print edition November, 2010

This book will interest researchers, scientists, engineers and graduate students in many disciplines, who make use of mathematical modeling and computer simulation. Although it represents only a small sample of the research activity on numerical simulations, the book will certainly serve as a valuable tool for researchers interested in getting involved in this multidisciplinary ïneld. It will be useful to encourage further experimental and theoretical researches in the above mentioned areas of numerical simulation.

\section{How to reference}

In order to correctly reference this scholarly work, feel free to copy and paste the following:

Shan-heng Yan, Qi Zhang, Na Zhao and Yong-gang Yu (2010). Experimental Investigation and Numerical Simulation on Interaction Process of Plasma Jet and working Medium, Numerical Simulations - Examples and Applications in Computational Fluid Dynamics, Prof. Lutz Angermann (Ed.), ISBN: 978-953-307-153-4, InTech, Available from: http://www.intechopen.com/books/numerical-simulations-examples-and-applications-incomputational-fluid-dynamics/experimental-investigation-and-numerical-simulation-on-interaction-process-ofplasma-jet-and-working

\section{INTECH}

open science | open minds

\section{InTech Europe}

University Campus STeP Ri

Slavka Krautzeka 83/A

51000 Rijeka, Croatia

Phone: +385 (51) 770447

Fax: +385 (51) 686166

www.intechopen.com

\section{InTech China}

Unit 405, Office Block, Hotel Equatorial Shanghai

No.65, Yan An Road (West), Shanghai, 200040, China

中国上海市延安西路65号上海国际贵都大饭店办公楼405单元

Phone: +86-21-62489820

Fax: +86-21-62489821 
(C) 2010 The Author(s). Licensee IntechOpen. This chapter is distributed under the terms of the Creative Commons Attribution-NonCommercialShareAlike-3.0 License, which permits use, distribution and reproduction for non-commercial purposes, provided the original is properly cited and derivative works building on this content are distributed under the same license. 